\title{
Modeling and Analysis of Protein Design under Resource Constraints
}

\author{
YUVAL NOV and LAWRENCE M. WEIN
}

\begin{abstract}
The potency, or fitness, of a protein-based drug can be enhanced by changing the sequence of its underlying protein. We present a novel stochastic model for the sequence-fitness relation, and estimate its four parameters from industrial data. Using this model, we formulate and analyze two variants of the protein design problem. In the single-period design problem, the designer needs to decide under capacity constraints which set of sequences to screen in order to maximize the expected fitness of the best sequence in the set. In the more general two-period design problem, the designer can afford two screening rounds and needs to allocate resources optimally across the two periods to maximize the same objective function. Analytical and simulation results allow us to assess the utility of the proposed design strategies for various parameter regimes.
\end{abstract}

Key words: protein design, fitness landscape, directed evolution, stochastic optimization.

\section{INTRODUCTION}

$\mathbf{P}$ ROTEINS ARE PERHAPS THE MOST IMPORTANT MOLECUlES in living organisms. Enzymes that catalyze biochemical reactions, antibodies that fight infectious diseases, hormones that regulate cells' behavior, and hemoglobins that transport oxygen in the blood are all examples of proteins, illustrating their versatility (Stryer, 1995). Because of their crucial role in virtually all physiological processes, proteins have become a huge industry. In recent years, it has become technologically possible to produce proteins in mass quantities and use them as pharmaceuticals (Walsh, 2002). Protein-based drug sales are estimated at $\$ 40$ billion in 2003 and are growing faster than sales of most other therapeutics (BCC Research, 2003). Other, nonpharmaceutical, products that are based on proteins include detergents and fertilizers.

Drug designers often try to improve the potency of a drug by enhancing one or more of its chemical properties. Antibodies, for example, need to bind tightly and quickly to their targets, and enzymes need to exhibit a high catalytic activity. The performance of a protein in achieving a chemical task can be measured quantitatively in a laboratory, and the result of such a measurement is called the protein's fitness. It is up to the designer to define exactly what is the task and how to measure fitness accordingly.

Changes in the molecular structure of a protein often result in changes of fitness; but how can such changes be made? Proteins are highly modular molecules, made of smaller building blocks-molecules called amino acids or residues, of which there are exactly 20 naturally occuring types. A protein is a folded sequence of residues, whose spatial structure and electrical properties determine its fitness; each position

Graduate School of Business, Stanford University, Stanford, CA 94305. 
along this sequence is called a site. Replacement of certain residues along the sequence by others-an act called mutation - often results in structural changes, and ultimately, in changes in fitness. The version of a protein typically found in nature is called the wild type, and mutated versions of the wild type are called variants

The systematic search of drug developers towards a high-fitness variant is called protein design. Although it is today technologically possible to produce virtually any desired protein variant, screening exhaustively all $20^{n}$ variants of a protein of length $n$ is impractical, as proteins are typically several hundreds of residues long. Protein design can therefore be viewed as a resource allocation problem: given capacity and time constraints, drug developers need to decide, often dynamically, which variants to produce and screen while trying to maximize fitness.

At the basis of protein design lies the mapping from a protein's sequence to its fitness. Although this mapping is deterministic and governed by the laws of physics, it is extremely complex. One approach to protein design, called rational design, is to gain sufficient understanding of the three-dimensional structure of a protein so that promising modifications to the protein sequence can be identified. While this approach has been fruitful, the rational design of proteins for specific functions appears to be years away. Consequently, many protein designers take an alternative route: they mimic nature by carrying out multiple rounds of mutation, recombination (i.e., combining segments of other proteins to form a new protein) and screening (i.e., testing for high fitness). Many technologies are available in the laboratory to perform these three operations in a high-volume manner (see, e.g., the first paragraph of Fox et al. [2003]). This general approach is referred to as directed evolution, and the strategies employed (e.g., which of the screened variants from one round should be used as starting points for the next round, which variants should be discarded, and what mutation and recombination rates should be used in each round) are heavily influenced by genetic algorithms and classical agricultural breeding techniques.

Not surprisingly, much work has been done on developing mathematical models of the sequence-fitness relationship, and on subsequent analysis of various directed evolution strategies. This field is too extensive to provide anything but the crudest overview here, and we refer readers to Voigt et al. (2001) for a thoughtful review of the literature. Wright (1932) introduced the concept of a fitness landscape in which each genetic sequence corresponds to a point in a multidimensional space and has a corresponding fitness value that measures the height of the landscape at this location; this idea was generalized to proteins by Maynard Smith (1970). According to this paradigm, directed evolution is visualized as an adaptive walk in this landscape that ideally ends up traversing a high peak. Several different classes of fitness landscape models exist. Eigen et al. (1989) use differential equation models that capture the kinetics of replication, degradation, and mutation and apply these models to RNA folding (Fontana and Schuster, 1998). Another approach quantifies the free energy in three-dimensional lattice models (e.g., Govindarajan and Goldstein, 1997). Both of these approaches are able to mimic several nontrivial features of RNA folding or protein folding.

As is often the case with highly complex systems, it is also beneficial to think of the sequence-fitness relation as a random phenomenon, which lends itself to a probabilistic analysis. That is, the fitness values of all possible sequences are viewed as a realization of a stochastic process. The two major research thrusts in this area revolve around the $N K$ model and the "rough Mt. Fuji" model. In the $N K$ model, first introduced by Kauffman and Levin (1987), the fitness of a protein is the sum of the fitness contributions from each of the protein's $N$ sites, and the fitness contribution of each site depends not only on the choice of residue at that site, but also on the choice of residues at $K$ other sites, which may be neighboring sites or chosen at random. By varying the value of $K$, the "ruggedness" of the landscape can be tuned. If $K=0$, then the landscape is smooth and contains a single optimum, and at the other extreme, $K=N-1$ and the model coincides with the random energy model (Derrida, 1981), which is very rugged, i.e., has many local optima. Aita and Husimi's rough Mt. Fuji model (Aita and Husimi, 2000a) is a nonstationary spatial model that measures fitness deviations from a mountain peak, and has a drift that represents the slope of the mountain. The fitness in their model can be decomposed into an additive part (i.e., the change in fitness due to a multisite mutation is roughly equal to the sum of the changes from the corresponding single-site mutations, as in the $K=0$ version of the $N K$ model) and a noise term to capture the nonadditivity. In both the $N K$ model and the rough Mt. Fuji model, certain directed evolution strategies can be modeled as adaptive walks on the fitness landscapes and can be analyzed probabilistically; Weinberger and colleagues (e.g., Weinberger, 1990) performed much of the early analysis of the $N K$ model, and Aita and Husimi 
have extensively analyzed variants of their model (e.g., Aita and Husimi, 2000b). In addition, Aita and colleagues (e.g., Aita et al., 2000, 2001) have empirically validated their model and found that it is a good fit in certain regions the of sequence space.

In this paper, we formulate a new model for the sequence-fitness relation, estimate its parameters from empirical data (Wu et al., 1998), and analyze several protein-design optimization problems that incorporate our fitness model. Like the $N K$ model (two parameters, $N$ and $K$ ) and the rough Mt. Fuji model (three main parameters, the mean slope, the altitude and the roughness), our model is parsimonious (four parameters) and somewhat analytically tractable. Like the rough Mt. Fuji model but in contrast to the $N K$ model, our model is spatially nonstationary and has a drift; whereas the rough Mt. Fuji model measures deviations from an unknown mountain peak, our model measures deviations from the wild type, which is the natural starting point in directed evolution studies. In addition, our model is similar to the rough Mt. Fuji model in that it assumes mutational additivity (the validity of this assumption is discussed in Section 2) plus a noise term to capture nonadditivity. The most distinctive aspect of our model relative to the Mt. Fuji model is that it captures the phenomenon (observed in the laboratory data of Wu et al. [1998]) that the majority of desirable variants are generated by mutations in just a few sites. While a variation of the $N K$ model could probably be constructed to capture this phenomenon, the resulting model would not capture the nonstationarity and associated drift and would likely be less analytically tractable than our model.

Our problem formulation also differs from those in the literature. First, motivated by current industrial practice, we impose constraints on the total number of variants screened and on the total amount of time; the considerable amount of time required to perform one round of screening, coupled with customers' expectations of short lead times, lead us to consider problems with either one or two screening rounds. Second, rather than choosing mutations randomly (from within a specified class) as is typically done, we specify the actual set of variants in each screening round. Although we have found only one dataset suitable for parameter estimation, the ultimate goal of this work is twofold: to empirically determine whether different types of proteins (e.g., antibodies, industrial enzymes) map into different parts of the four-dimensional parameter space, and to determine promising strategies for different regions of parameter space.

In Section 2, we formulate the model and estimate its parameters from empirical data. We study in Sections 3 and 4 two types of problems a drug designer may face: the single-period design problem and the two-period design problem. In the latter problem, the fitness values observed in the first period are used to choose the variants screened in the second period. The difficulty of these two decision problems precludes the derivation of exact optimal solutions, and we resort to a variety of bounds, approximations, and heuristics, and ultimately to simulation. We offer concluding remarks in Section 5.

\section{MODELING PROTEIN FITNESS}

After stating in Section 2.1 the protein characteristics that our model attempts to capture, we formulate the model in Section 2.2. A distributional analysis of the model is performed in Section 2.3, which is required for the parameter estimation task in Section 2.4 and for the analysis of the optimization problems in the subsequent sections.

\subsection{Requirements from the model}

Our model attempts to capture three characteristics of proteins, which are listed below.

Dominance in mean of the wild type. It is known that a priori, mutating the wild type is more likely to decrease fitness than to increase it. Also, the more "different" a sequence is from the wild-type sequence (we define a distance function between sequences later), the lower its fitness is, on average (Voigt et al., 2001).

Mutational additivity. A fitness landscape is said to be additive if the change in fitness caused by a multiple-site mutation approximately equals the sum of the fitness changes caused by the corresponding single-site mutations. While certainly not all proteins are highly additive, many proteins exhibit at least moderate additivity (see Wells [1990] and Voigt et al. [2001, Section A.2] for a detailed discussion), and we restrict our modeling and analysis below to the latter. In our model, it is enough that the location and 
extent of nonadditivity will be difficult to predict a priori, so that it is most appropriately captured by noise terms.

Clustering. When a substitution of the wild-type residue at a site is known to increase fitness, other substitutions at the same site are more likely to increase fitness as well. Thus, favorable mutations tend to cluster in relatively few sites (see Appendix A for details).

\subsection{The Model}

Let $R$ be the alphabet of all 20 residues, denoted by their standard one-letter abbreviations $(\mathrm{A}=$ Alanine, C $=$ Cysteine, etc.; see Stryer [1995]), and consider a protein made of $n$ residues. We define $\mathscr{S}=\left\{\left(r_{1}, \ldots, r_{n}\right): r_{i} \in R\right\}$ to be the sequence space - the set of all $n$-tuples of residues-and denote the wild-type sequence by $\hat{s}=\left(\hat{r}_{1}, \ldots, \hat{r}_{n}\right)$. We also let $F_{s}$ be the fitness of a sequence $s \in \mathscr{S}$ and define $\mathbf{F}=\left(F_{s}, s \in \mathscr{S}\right)$.

To build a probabilistic model for protein fitness, we need to specify the distribution of the random vector $\mathbf{F}$. We now do so and construct a multivariate normal model for $\mathbf{F}$, which depends on four landscape parameters: the drift $m$, the residue variance $\sigma_{\mathrm{R}}^{2}$, the site variance $\sigma_{\mathrm{S}}^{2}$, and the nonadditivity variance $\sigma_{\mathrm{N}}^{2}$.

By mutating at site $i$ and replacing the residue $\hat{r}_{i}$ with $r \neq \hat{r}_{i}$, we contribute a quantity $f_{i}(r) \sim N\left(\mu_{i}, \sigma_{\mathrm{R}}^{2}\right)$ to the overall fitness. Note that the mean $\mu_{i}$ of $f_{i}(r)$ depends on the site $i$. When $i \neq i^{\prime}$, the random variables $f_{i}(r)$ and $f_{i^{\prime}}\left(r^{\prime}\right)$ are independent; for each $i$, we model the 19 random variables $f_{i}(r), r \neq \hat{r}_{i}$, as independent of each other given $\mu_{i}$. However, we also model $\mu_{1}, \ldots, \mu_{n}$ as independent random variables, having an $N\left(m, \sigma_{\mathrm{S}}^{2}\right)$ distribution with mean $m<0$. Finally, we set the fitness of the wild type to zero (without loss of generality) and define the fitness of any other sequence $s=\left(r_{1}, \ldots, r_{n}\right)$ to be

$$
F_{s}=\sum_{i: r_{i} \neq \hat{r}_{i}} f_{i}\left(r_{i}\right)+\epsilon_{s}
$$

where the summation ranges over all mutated sites, and the $\epsilon_{s}, s \in \mathscr{S}$, are $N\left(0, \sigma_{\mathrm{N}}^{2}\right)$ noise terms, independent of each other and of any other quantity mentioned thus far.

In Section 2.3, we analyze the distribution of $\mathbf{F}$ in full detail; however, we can already see, informally, how the model captures the three aforementioned characteristics: by setting the drift $m$ to be negative, we get that $E\left[f_{i}(r)\right]<0$, so that mutations decrease fitness, on average; by making $F_{s}$ roughly the sum of the fitness contributions $f_{i}\left(r_{i}\right)$ in (1), we obtain mutational additivity and may quantify the nonadditivity via the nonadditivity variance $\sigma_{\mathrm{N}}^{2}$; and by making, for each $i$, the 19 random variables $f_{i}(r), r \neq \hat{r}_{i}$ depend on a common $\mu_{i}$, we establish positive correlation among them and capture the clustering phenomenon. Note that the degree of clustering is expected to be large when the site variance $\sigma_{\mathrm{S}}^{2}$ is greater than the residue variance $\sigma_{\mathrm{R}}^{2}$.

The model can be modified in several straightforward ways. Proteins usually interact with other molecules through special sites, called active sites (Stryer, 1995). These sites are the most dominant in determining fitness, and it is therefore better to focus mutation efforts at them (Wu et al., 1998). We can incorporate this in our model by redefining the protein length $n$ and the sequence space $\mathscr{S}$ accordingly. In addition, the number of possible substitutions at each site may also differ from that described above. The 20 naturally occuring residues can be classified into groups in various ways (e.g., hydrophobic/hydrophilic), and it is sometimes enough to consider residues from only one group when mutating a site; on the other hand, drug developers sometimes experiment with artificial residues that do not occur in nature (Anthony-Cahill and Magliery, 2002), in which case there are more than 19 substitutions at each site. Our model can cover these modifications by redefining the alphabet $R$. Although the size of $\mathscr{S}$ may be significantly reduced as a result of the above, it is still enormous, so the motivation for our work is not diminished.

\subsection{Distributional Analysis}

A prerequisite for the parameter estimation in Section 2.4 and for the analysis of the optimization problems in the subsequent sections is a distributional analysis, which we perform in this subsection. Let $\boldsymbol{\mu}$ and $\boldsymbol{\Sigma}$ denote the mean vector and covariance matrix of $\mathbf{F}$, so that $\mathbf{F} \sim N(\boldsymbol{\mu}, \boldsymbol{\Sigma})$. To spell out the elements of $\boldsymbol{\mu}$ and $\boldsymbol{\Sigma}$, we reexpress the model $\mathbf{F}$ using independent building blocks as follows. 
The normal random variable $f_{i}(r) \sim N\left(\mu_{i}, \sigma_{\mathrm{R}}^{2}\right)$ having the random mean $\mu_{i} \sim N\left(m, \sigma_{\mathrm{S}}^{2}\right)$ can be thought of as the sum

$$
f_{i}(r)=\mu_{i}+\tilde{f}_{i}(r), \quad r \neq \hat{r}_{i},
$$

where the zero-mean $\tilde{f}_{i}(r) \sim N\left(0, \sigma_{\mathrm{R}}^{2}\right)$ is independent of the random variable $\mu_{i}$. Consequently, the fitness of a sequence $s=\left(r_{1}, \ldots, r_{n}\right)$ can be written as

$$
F_{s}=\sum_{i: r_{i} \neq \hat{r}_{i}}\left(\mu_{i}+\tilde{f}_{i}\left(r_{i}\right)\right)+\epsilon_{s}, \quad s \neq \hat{s},
$$

where the building blocks

$$
\mu_{i}, i=1, \ldots, n ; \quad \tilde{f}_{i}(r), i=1, \ldots, n, r \neq \hat{r}_{i} ; \quad \epsilon_{s}, s \in \mathscr{S}
$$

are all independent of each other.

It is easier to derive the distribution of $\mathbf{F}$ using the representation (2) rather than (1). Let $d\left(s, s^{\prime}\right)$ denote the Hamming distance (Hamming, 1950) between the two sequences $s=\left(r_{1}, \ldots, r_{n}\right)$ and $s^{\prime}=\left(r_{1}^{\prime}, \ldots, r_{n}^{\prime}\right)$ in $\mathscr{S}$; this distance is the number of sites in which $s$ and $s^{\prime}$ differ, i.e.,

$$
d\left(s, s^{\prime}\right)=\left|\left\{i: r_{i} \neq r_{i}^{\prime}\right\}\right| \text {. }
$$

For example,

$$
s=(\mathrm{C}, \mathrm{A}, \mathrm{E}, \mathrm{A}), s^{\prime}=(\mathrm{D}, \mathrm{A}, \mathrm{E}, \mathrm{F}) \quad \Rightarrow \quad d\left(s, s^{\prime}\right)=2
$$

Then it follows that

$$
\begin{aligned}
E\left[F_{s}\right] & =E\left[\sum_{i: r_{i} \neq \hat{r}_{i}}\left(\mu_{i}+\tilde{f}_{i}\left(r_{i}\right)\right)+\epsilon_{s}\right] \\
& =\sum_{i: r_{i} \neq \hat{r}_{i}}\left(E\left[\mu_{i}\right]+E\left[\tilde{f}_{i}(r)\right]\right)+E\left[\epsilon_{s}\right] \\
& =d(s, \hat{s}) m .
\end{aligned}
$$

Indeed, the mean fitness of a sequence is a decreasing function of its distance from the wild type $\hat{s}$ (recall that the drift $m$ is negative), and it is always lower than the wild type's zero fitness.

To express the covariance between the fitness values of two variants, we define two types of sets as follows. For two sequences $s, s^{\prime} \in \mathscr{S}$, let $M_{1}\left(s, s^{\prime}\right)$ be the set of sites in which both $s$ and $s^{\prime}$ differ from the wild type; let $M_{2}\left(s, s^{\prime}\right)$ be the set of sites in which $s$ and $s^{\prime}$ have the same residue, and this residue is different from the corresponding wild-type residue. More tersely,

$$
M_{1}\left(s, s^{\prime}\right)=\left\{i: r_{i}, r_{i}^{\prime} \neq \hat{r}_{i}\right\} \quad \text { and } \quad M_{2}\left(s, s^{\prime}\right)=\left\{i: r_{i}=r_{i}^{\prime} \neq \hat{r}_{i}\right\}
$$

For example, if the wild-type sequence is $\hat{s}=(\mathrm{A}, \mathrm{A}, \mathrm{A}, \mathrm{A})$ and $s$ and $s^{\prime}$ are as in (4), then

$$
M_{1}\left(s, s^{\prime}\right)=\{1,3\} \quad \text { and } \quad M_{2}\left(s, s^{\prime}\right)=\{3\} .
$$

Recalling that the building blocks in (3) are all independent, we may write for $s \neq s^{\prime}$

$$
\begin{aligned}
\operatorname{Cov}\left(F_{s}, F_{s^{\prime}}\right) & =\operatorname{Cov}\left(\sum_{i: r_{i} \neq \hat{r}_{i}}\left(\mu_{i}+\tilde{f}_{i}\left(r_{i}\right)\right)+\epsilon_{s}, \sum_{i: r_{i}^{\prime} \neq \hat{r}_{i}}\left(\mu_{i}+\tilde{f}_{i}\left(r_{i}^{\prime}\right)\right)+\epsilon_{s^{\prime}}\right) \\
& =\sum_{i \in M_{1}\left(s, s^{\prime}\right)} \operatorname{Var}\left(\mu_{i}\right)+\sum_{i \in M_{2}\left(s, s^{\prime}\right)} \operatorname{Var}\left(\tilde{f}_{i}(r)\right) \\
& =\sigma_{\mathrm{S}}^{2}\left|M_{1}\left(s, s^{\prime}\right)\right|+\sigma_{\mathrm{R}}^{2}\left|M_{2}\left(s, s^{\prime}\right)\right| .
\end{aligned}
$$


To find the fitness variance of a sequence $s$, we note that $d(s, \hat{s})=\left|M_{1}(s, s)\right|=\left|M_{2}(s, s)\right|$, which gives

$$
\begin{aligned}
\operatorname{Var}\left(F_{S}\right) & =\operatorname{Cov}\left(F_{s}, F_{s}\right) \\
& =\operatorname{Cov}\left(\sum_{i: r_{i} \neq \hat{r}_{i}}\left(\mu_{i}+\tilde{f}_{i}\left(r_{i}\right)\right)+\epsilon_{s}, \sum_{i: r_{i} \neq \hat{r}_{i}}\left(\mu_{i}+\tilde{f}_{i}\left(r_{i}\right)\right)+\epsilon_{s}\right) \\
& =\sum_{i \in M_{1}(s, s)} \operatorname{Var}\left(\mu_{i}\right)+\sum_{i \in M_{2}(s, s)} \operatorname{Var}\left(\tilde{f}_{i}(r)\right)+\operatorname{Var}\left(\epsilon_{s}\right) \\
& =d(s, \hat{s})\left(\sigma_{\mathrm{S}}^{2}+\sigma_{\mathrm{R}}^{2}\right)+\sigma_{\mathrm{N}}^{2} .
\end{aligned}
$$

We summarize the above as

$$
\begin{aligned}
E\left[F_{s}\right] & =d(s, \hat{s}) m, \\
\operatorname{Var}\left(F_{s}\right) & =d(s, \hat{s})\left(\sigma_{\mathrm{S}}^{2}+\sigma_{\mathrm{R}}^{2}\right)+\sigma_{\mathrm{N}}^{2}, \quad s \neq \hat{s}, \\
\operatorname{Cov}\left(F_{s}, F_{s^{\prime}}\right) & =\sigma_{\mathrm{S}}^{2}\left|M_{1}\left(s, s^{\prime}\right)\right|+\sigma_{\mathrm{R}}^{2}\left|M_{2}\left(s, s^{\prime}\right)\right| .
\end{aligned}
$$

Since our model is Gaussian, we may use well-known formulas (Tong, 1990) to compute conditional distributions. Suppose that $\mathbf{F}, \boldsymbol{\mu}$, and $\boldsymbol{\Sigma}$ are partitioned as

$$
\mathbf{F}=\left(\begin{array}{l}
\mathbf{F}_{1} \\
\mathbf{F}_{2}
\end{array}\right), \quad \boldsymbol{\mu}=\left(\begin{array}{l}
\boldsymbol{\mu}_{1} \\
\boldsymbol{\mu}_{2}
\end{array}\right), \quad \text { and } \quad \boldsymbol{\Sigma}=\left(\begin{array}{ll}
\boldsymbol{\Sigma}_{11} & \boldsymbol{\Sigma}_{12} \\
\boldsymbol{\Sigma}_{21} & \boldsymbol{\Sigma}_{22}
\end{array}\right)
$$

Then, upon observing the fitness values of the subvector $\mathbf{F}_{1}$, we can update the fitness distribution of the remaining variants in $\mathbf{F}_{2}$ by

$$
E\left[\mathbf{F}_{2} \mid \mathbf{F}_{1}\right]=\boldsymbol{\mu}_{2}+\boldsymbol{\Sigma}_{21} \boldsymbol{\Sigma}_{11}^{-1}\left(\mathbf{F}_{1}-\boldsymbol{\mu}_{1}\right) \quad \text { and } \quad \operatorname{Cov}\left(\mathbf{F}_{2} \mid \mathbf{F}_{1}\right)=\boldsymbol{\Sigma}_{22}-\boldsymbol{\Sigma}_{21} \boldsymbol{\Sigma}_{11}^{-1} \boldsymbol{\Sigma}_{12}
$$

Note that the conditional covariance does not depend on the observed values $\mathbf{F}_{1}$, a property we shall use later.

\subsection{Parameter Estimation}

In this subsection, we estimate the landscape parameters from data obtained during the design of the drug Vitaxin (Wu et al., 1998). Vitaxin is a monoclonal antibody that is intended to block the proliferation of new blood vessels surrounding cancerous tumors. As a measure of fitness of the sequence $s$, we chose $\log _{10} K_{\mathrm{d}}(\hat{s})-\log _{10} K_{\mathrm{d}}(s)$, where $K_{\mathrm{d}}(s)$ is the ratio between the dissociation rate constant $k_{\text {off }}$ and the association rate constant $k_{\text {on }}$ of the sequence $s$. Since the data is censored, we use the expectation maximization (EM) algorithm (McLachlan and Krishnan, 1997) to estimate the landscape parameters, and obtain

$$
\hat{m}=-1.35, \quad \hat{\sigma}_{\mathrm{S}}^{2}=0.25, \quad \hat{\sigma}_{\mathrm{N}}^{2}=0.14, \quad \hat{\sigma}_{\mathrm{R}}^{2}=0.18
$$

The full details of the derivation of these parameters are given in Appendix B.

These estimated values are consistent with the characteristics we attempted to capture: the estimate of the drift $m$ is negative; the estimate of the nonadditivity variance $\sigma_{\mathrm{N}}^{2}$ is small relative to the other estimated variance parameters, supporting additivity; and the estimate of the site variance $\sigma_{\mathrm{S}}^{2}$ is larger than the residue variance $\sigma_{\mathrm{R}}^{2}$, in accordance with the clustering phenomenon. In addition, a goodness-of-fit test yields a $p$-value of 0.51, providing no evidence to reject our model (see Appendix B.7 for details). 


\section{THE SINGLE-PERIOD DESIGN PROBLEM}

Equipped with a model for protein fitness, we can model the decision problems drug designers are facing. Producing and screening protein variants is both expensive and time consuming. Suppose we have enough resources to produce and screen only $K$ variants, and enough time to afford only one production and screening round. We then need to choose a subset $D \subseteq \mathscr{S}$ of sequences that we call a design, produce and screen its variants to observe their fitness values, and keep the one with the highest fitness. Our goal is, therefore, to find a design that will maximize the expected fitness achieved in this process, i.e., to solve

$$
\underset{D \subseteq \mathscr{S}}{\operatorname{maximize}} E\left[\max _{s \in D} F_{S}\right] \quad \text { s.t. }|D| \leq K .
$$

Clearly, the constraint should hold with equality in the optimal solution. To simplify notation, we define

$$
F_{D}=\max _{s \in D} F_{S}, \quad D \subseteq \mathscr{S}
$$

and call $F_{D}$ the fitness of the design $D$.

We assume here that the drug designer knows only the value of $K$ and the values of the landscape parameters $m, \sigma_{\mathrm{S}}^{2}, \sigma_{\mathrm{R}}^{2}$, and $\sigma_{\mathrm{N}}^{2}$ before choosing the design $D$. We also assume that $D$ does not contain the wild-type sequence $\hat{s}$ (although $\hat{s}$ technically belongs to the sequence space $\mathscr{S}$ ), as its fitness is known in advance to be zero. The problem in (12) is called "the single-period design problem" because it involves only one round of production and screening; we discuss the more general two-period problem in Section 4.

The remainder of this section is devoted to analyzing (12). In Section 3.1 we derive, and approximately evaluate, upper and lower bounds on $E\left[F_{D}\right]$. We tackle a special case in Section 3.2, where all sequences have a single-point mutation. In Section 3.3, we provide motivation for a heuristic that chooses a design to minimize its total covariance, a quantity that is related to the upper bound in Section 3.1. Finally, a general heuristic procedure for choosing a single-period design is constructed in Section 3.4.

\subsection{Upper and lower fitness bounds}

The analysis of (12) is intimately related to the distribution of the random variable $F_{D}$ in (13). When $D$ is a general design, the distribution of $F_{D}$ is essentially the distribution of the maximal element in a general multivariate normal vector. Unfortunately, the analysis of this latter distribution is difficult, and to the best of our knowledge, only when the size of the vector is as low as two are there known closed-form formulas for the first several moments thereof (Clark, 1961; Kella, 1986). To retain tractability, we restrict the design $D$ to contain sequences all having the same number of mutations.

To this end, we define a $p$-mutant to be a sequence that differs from the wild type in exactly $p$ out of its $n$ sites. We define $\mathscr{S}_{p}=\{s \in \mathscr{S}: d(s, \hat{s})=p\}$ to be the set of all $p$-mutants, and let $\mathscr{D}_{K}(p)=\left\{D \subseteq \mathscr{S}_{p}:|D|=K\right\}$ denote the collection of all designs of size $K$ made of only $p$-mutants. It follows from (6) and (7) that for fixed $p$, the random variables $F_{s}, s \in \mathscr{S}_{p}$, all share the same marginal distribution with

$$
E\left[F_{s}\right]=p m, \quad \operatorname{Var}\left(F_{s}\right)=p\left(\sigma_{\mathrm{S}}^{2}+\sigma_{\mathrm{R}}^{2}\right)+\sigma_{\mathrm{N}}^{2}, \quad s \in \mathscr{S}_{p}
$$

Although the covariance between $F_{s}$ and $F_{s^{\prime}}, s, s^{\prime} \in \mathscr{S}_{p}$, may take many values, it follows from (8) that it is always nonnegative. We use these observations to stochastically bound $F_{D}, D \in \mathscr{D}_{K}(p)$ from above and below and then approximately evaluate these bounds.

We first derive a stochastic upper bound, $U_{K}(p)$, on the random variable $F_{D}$ when $D \in \mathscr{D}_{K}(p)$. Fix $p$, and let $G_{1}, G_{2}, \ldots$ be independent normal random variables, all distributed in the same way as $F_{S}$ in (14):

$$
E\left[G_{i}\right]=p m, \quad \operatorname{Var}\left(G_{i}\right)=p\left(\sigma_{\mathrm{S}}^{2}+\sigma_{\mathrm{R}}^{2}\right)+\sigma_{\mathrm{N}}^{2}, \quad \operatorname{Cov}\left(G_{i}, G_{j}\right)=0 \quad \text { for } i \neq j .
$$

If we define $G^{(K)}=\max \left\{G_{1}, \ldots, G_{K}\right\}$, then it follows from Slepian's inequality (see Appendix C) that $G^{(K)}$ stochastically dominates $F_{D}$, i.e.,

$$
P\left(F_{D}>x\right) \leq P\left(G^{(K)}>x\right), \quad x \in \mathbb{R}, D \in \mathscr{D}_{K}(p),
$$


which in turn implies

$$
E\left[F_{D}\right] \leq E\left[G^{(K)}\right], \quad D \in \mathscr{D}_{K}(p)
$$

Note that these bounds are uniform in the sense that they are independent of the choice of $D$, as long as $D \in \mathscr{D}_{K}(p)$.

Because our ultimate goal is to estimate $E\left[F_{D}\right]$, we use extreme value theory to approximate the values of $P\left(G^{(K)}>x\right)$ and $E\left[G^{(K)}\right]$. More specifically, it is known that under the right normalization, the distribution of the maximum of $K$ independent and identically distributed normal random variables converges in distribution to a Gumbel random variable as $K \rightarrow \infty$ (see Appendix D). Thus, for large $K$, the distribution of $G^{(K)}$ approximately equals that of a Gumbel random variable, which we denote by $U_{K}(p)$, having the parameters

$$
\alpha=\frac{a_{K}}{\sqrt{\sigma_{\mathrm{N}}^{2}+p\left(\sigma_{\mathrm{S}}^{2}+\sigma_{\mathrm{R}}^{2}\right)}} \quad \text { and } \quad \beta=b_{K} \sqrt{\sigma_{\mathrm{N}}^{2}+p\left(\sigma_{\mathrm{S}}^{2}+\sigma_{\mathrm{R}}^{2}\right)}+m p
$$

where the normalizing constants

$$
a_{K}=\sqrt{2 \log K}, \quad b_{K}=\sqrt{2 \log K}-\frac{\log \log K+\log 4 \pi}{2 \sqrt{2 \log K}}
$$

depend only on $K$. Hence, for large $K$ and $D \in \mathscr{D}_{K}(p)$, we can write

$$
\begin{aligned}
P\left(F_{D}>x\right) & \leq P\left(G^{(K)}>x\right) \\
& \approx P\left(U_{K}(p)>x\right) \\
& =1-\exp \left\{-\exp \left\{-a_{K}\left(\frac{x-p m}{\sqrt{\sigma_{\mathrm{N}}^{2}+p\left(\sigma_{\mathrm{S}}^{2}+\sigma_{\mathrm{R}}^{2}\right)}}-b_{K}\right)\right\}\right\},
\end{aligned}
$$

and

$$
E\left[F_{D}\right] \leq E\left[G^{(K)}\right] \approx E\left[U_{K}(p)\right]=p m+\sqrt{\sigma_{\mathrm{N}}^{2}+p\left(\sigma_{\mathrm{S}}^{2}+\sigma_{\mathrm{R}}^{2}\right)}\left(b_{K}+\frac{\gamma}{a_{K}}\right),
$$

where $\gamma=0.5772 \ldots$ is Euler's constant.

Using a slightly more sophisticated approach, we also derive a stochastic lower bound $L_{K}(p)$ on $F_{D}$ when $D \in \mathscr{D}_{K}(p)$. The maximal covariance between the fitness of two $p$-mutants is achieved when the two sequences have their mutations in the same $p$ sites, and in addition, have the same residue in $p-1$ of these sites. By (8), the covariance in this case is $v^{2} \triangleq \sigma_{\mathrm{S}}^{2} p+\sigma_{\mathrm{R}}^{2}(p-1)$. Let $\mathbf{W}$ be a $K \times K$ matrix having as diagonal terms the fitness variance of a $p$-mutant (namely, $p\left(\sigma_{\mathrm{S}}^{2}+\sigma_{\mathrm{R}}^{2}\right)+\sigma_{\mathrm{N}}^{2}$; see (7)), and as off-diagonal terms, $v^{2}$. Also, let $\boldsymbol{\mu}_{p}$ be the constant $K$-vector $(m p, \ldots, m p)^{\mathrm{T}}$ (the superscript $\mathrm{T}$ denotes transposition). Now define the random variables

$$
\left(H_{1}, \ldots, H_{K}\right)^{\mathrm{T}} \sim N\left(\boldsymbol{\mu}_{p}, \mathbf{W}\right), \quad H^{(K)}=\max \left\{H_{1}, \ldots, H_{K}\right\} .
$$

It follows again from Slepian's inequality that if $D \in \mathscr{D}_{K}(p)$ then

$$
P\left(F_{D}>x\right) \geq P\left(H^{(K)}>x\right), \quad x \in \mathbb{R}, \quad D \in \mathscr{D}_{K}(p),
$$

which implies

$$
E\left[F_{D}\right] \geq E\left[H^{(K)}\right]
$$

Again, these bounds are independent of the choice of $D$ as long as $D \in \mathscr{D}_{K}(p)$. 
We cannot approximate the distribution of $H^{(K)}$ in the same way we approximated the distribution of $G^{(K)}$ earlier, since the random variables $H_{1}, \ldots, H_{K}$ are correlated. To circumvent this difficulty, we note that

$$
H^{(K)} \stackrel{D}{=} \widetilde{H}_{0}+\max \left\{\widetilde{H}_{1}, \ldots, \widetilde{H}_{K}\right\}
$$

where $\stackrel{D}{=}$ denotes equality in distribution,

$$
\tilde{H}_{0} \sim N\left(m p, v^{2}\right), \quad \widetilde{H}_{1}, \ldots, \widetilde{H}_{K} \sim N\left(0, \sigma_{\mathrm{R}}^{2}+\sigma_{\mathrm{N}}^{2}\right),
$$

and the $K+1$ random variables $\widetilde{H}_{0}, \widetilde{H}_{1}, \ldots, \widetilde{H}_{K}$ are all independent. Using again the convergence of the maximal normal to a Gumbel, we have that for large $K$, the distribution of the random variable $\max \left\{\widetilde{H}_{1}, \ldots, \widetilde{H}_{K}\right\}$ approximately equals that of a Gumbel random variable $\Gamma^{(K)}$ with parameters (see again Appendix D)

$$
\alpha=\frac{a_{K}}{\sqrt{\sigma_{\mathrm{R}}^{2}+\sigma_{\mathrm{N}}^{2}}} \quad \text { and } \quad \beta=b_{K} \sqrt{\sigma_{\mathrm{R}}^{2}+\sigma_{\mathrm{N}}^{2}} \text {. }
$$

Letting $L_{K}(p)=\widetilde{H}_{0}+\Gamma^{(K)}$, we get from all of the above that when $D \in \mathscr{D}_{K}(p)$,

$$
\begin{aligned}
P\left(F_{D}>x\right) & \geq P\left(H^{(K)}>x\right) \\
& =P\left(\widetilde{H}_{0}+\max \left\{\widetilde{H}_{1}, \ldots, \widetilde{H}_{K}\right\}>x\right) \\
& \approx P\left(\widetilde{H}_{0}+\Gamma^{(K)}>x\right) \\
& =P\left(L_{K}(p)>x\right) .
\end{aligned}
$$

By independence, the density function of $L_{K}(p)$ is the convolution of the densities of $\widetilde{H}_{0}$ and $\Gamma^{(K)}$, both easily expressed in closed form. Therefore, we can evaluate the distribution function of $L_{K}(p)$ by numerical integration. The bounds in (15) and (17) will not be used hereafter, but they would be useful if the objective function was expressed in terms of the right tail of the design fitness (as it may be in some industrial settings) as opposed to the mean design fitness.

To bound $E\left[F_{D}\right]$ from below when $D \in \mathscr{D}_{K}(p)$, we have

$$
\begin{aligned}
E\left[F_{D}\right] & \geq E\left[H^{(K)}\right] \\
& =E\left[\widetilde{H}_{0}\right]+E\left[\max \left\{\widetilde{H}_{1}, \ldots, \widetilde{H}_{K}\right\}\right] \\
& \approx E\left[L_{K}(p)\right] \\
& =m p+\sqrt{\sigma_{\mathrm{R}}^{2}+\sigma_{\mathrm{N}}^{2}}\left(b_{K}+\frac{\gamma}{a_{K}}\right) .
\end{aligned}
$$

Because $K$ will typically be large in practice, we now have excellent approximations for uniform upper and lower bounds on the distribution function and the mean of $F_{D}$ for $D \in \mathscr{D}_{K}(p)$. We shall use these bounds in Section 3.4.

\subsection{Optimal Choice of 1-mutants}

Suppose we restrict ourselves to only 1-mutants when solving (12); i.e., we solve

$$
\underset{D \subseteq \mathscr{S}_{1}}{\operatorname{maximize}} E\left[\max _{s \in D} F_{S}\right] \quad \text { s.t. }|D| \leq K .
$$

This problem is fully tractable, and can be solved via majorization (see Appendix E) as follows. Let $D$ be a design of size $K$ made of only 1 -mutants (i.e., $\left.D \in \mathscr{D}_{K}(1)\right)$, and let the $i$ th component of $\mathbf{k}=\left(k_{1}, \ldots, k_{n}\right)$ 
denote the number of sequences in $D$ that have their mutation at site $i$, so that $\sum_{1}^{n} k_{i}=K$. It follows from (7) and (8) that the covariance matrix of $\left(F_{s}, s \in D\right)$ has the block form

$$
\boldsymbol{\Sigma}(\mathbf{k})=\left(\begin{array}{cccc}
\boldsymbol{\Sigma}_{1} & \mathbf{0} & \cdots & \mathbf{0} \\
\mathbf{0} & \boldsymbol{\Sigma}_{2} & \cdots & \mathbf{0} \\
& \cdots & & \\
\mathbf{0} & \mathbf{0} & \cdots & \boldsymbol{\Sigma}_{n}
\end{array}\right)
$$

where $\Sigma_{i}$ is a $k_{i} \times k_{i}$ matrix, having $\sigma_{\mathrm{S}}^{2}+\sigma_{\mathrm{R}}^{2}+\sigma_{\mathrm{N}}^{2}$ on its diagonal and $\sigma_{\mathrm{S}}^{2}$ off of it. Let $D^{\prime}$ be another design in $\mathscr{D}_{K}(1)$ and define $\mathbf{k}^{\prime}$ and $\boldsymbol{\Sigma}\left(\mathbf{k}^{\prime}\right)$ similarly. By (6), both $\left(F_{s}, s \in D\right)$ and $\left(F_{s}, s \in D^{\prime}\right)$ then have the same $K$-vector $(m, \ldots, m)^{\mathrm{T}}$ as their mean vector.

A result due to Tong (1988) states that if $\mathbf{k}$ majorizes $\mathbf{k}^{\prime}$ (see Appendix E), then $F_{D}$ is stochastically dominated by $F_{D^{\prime}}$, so that

$$
P\left(F_{D}>x\right) \leq P\left(F_{D^{\prime}}>x\right), \quad x \in \mathbb{R}, \quad \text { and } \quad E\left[F_{D}\right] \leq E\left[F_{D^{\prime}}\right] .
$$

Therefore, an optimal solution to (19) is a design $D^{*}$ that is majorized by all $D \in \mathscr{D}_{K}(1)$. It follows from the definition of majorization that such a design spreads the mutations maximally across the sites in the sense that it makes the components of the corresponding $\mathbf{k}^{*}$ as nearly equal as possible. When $n$ divides $K$, we have $\mathbf{k}^{*}=(K / n, \ldots, K / n)$; more generally, we need to make sure that $\left|k_{i}-k_{j}\right| \leq 1$ for all $i, j$. Note that this result is independent of the landscape parameters: no matter what the values of $m, \sigma_{\mathrm{S}}^{2}, \sigma_{\mathrm{R}}^{2}$, and $\sigma_{\mathrm{N}}^{2}$ are, the optimal solutions of (19) are the same.

For example, if the wild-type sequence is $\hat{s}=(\mathrm{A}, \mathrm{A}, \mathrm{A})$ and $K=7$, then $D^{*}$ may consist of the sequences

$\begin{array}{lll}(C, A, A) & (A, H, A) & (A, A, E) \\ (Y, A, A) & (A, F, A) & (A, A, D), \\ (H, A, A) & & \end{array}$

although this $D^{*}$ is not the only optimal solution in this example.

We can also characterize the optimal solutions of (19) in a different way: a design $D$ is optimal if and only if it minimizes the sum of the entries of the corresponding covariance matrix, which we call the total covariance of $D$ and denote by $\tau(D)$. To see this, observe that the sum of the entries of $\boldsymbol{\Sigma}(\mathbf{k})$ in (20) is

$$
\tau(D)=\sigma_{\mathrm{S}}^{2} \sum_{i=1}^{n} k_{i}^{2}+K\left(\sigma_{\mathrm{R}}^{2}+\sigma_{\mathrm{N}}^{2}\right)
$$

The last term in this expression is constant and $\sigma_{\mathrm{S}}^{2}$ is positive, so to minimize $\tau(D)$ we need to minimize $\sum_{1}^{n} k_{i}^{2}$. This is done by ensuring that $\left|k_{i}-k_{j}\right| \leq 1$ for all $i$ and $j$, for if there were $i$ and $j$ such that $k_{i}-k_{j} \geq 2$, we could subtract 1 from $k_{i}$ and add 1 to $k_{j}$, and decrease $\sum_{1}^{n} k_{i}^{2}$ by at least 2 . Since the condition $\left|k_{i}-k_{j}\right| \leq 1$ for all $i$ and $j$ amounts exactly to spreading the mutations maximally across the sites, we see that the optimal designs are exactly the ones that minimize total covariance. We shall use this observation in the following subsection.

\subsection{Covariance-minimizing designs of p-mutants}

Consider now a more general form of (19), where we allow using only $p$-mutants for some fixed $p$. The optimization problem is now

$$
\underset{D \subseteq \mathscr{S}_{p}}{\operatorname{maximize}} E\left[\max _{s \in D} F_{s}\right] \quad \text { s.t. } \quad|D| \leq K .
$$

Inspired by the optimality of the "minimizing total covariance" principle for the case $p=1$, we propose to adopt it as a heuristic for general $p$, that is, to choose as a solution of (22) a design whose total covariance 


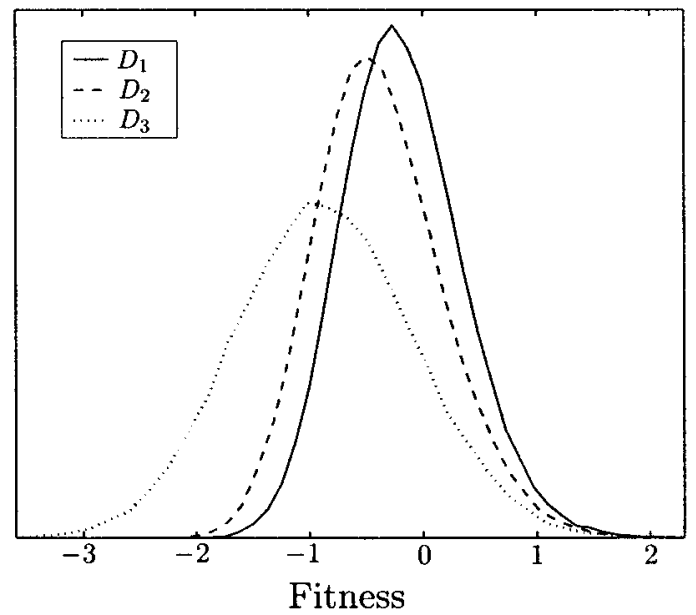

FIG. 1. Simulated distributions of $F_{D}$ for three designs in $\mathscr{D}_{200}(2)$. Total covariance values are $\tau\left(D_{1}\right)=3983$, $\tau\left(D_{2}\right)=10,479$, and $\tau\left(D_{3}\right)=20,876$. Landscape parameters are as in (11), and $n=10$.

is minimal among all the designs in $\mathscr{D}_{K}(p)$. Indeed, simulation results in Fig. 1 seem to indicate that the lower the total covariance $\tau(D)$ for $D \in \mathscr{D}_{K}(p)$ is, the higher is the expected fitness $E\left[F_{D}\right]$.

In Appendix F, we formulate the total-covariance minimization problem as an integer program and solve it in a strong sense: we show there how to build, for each $p$, an ordered list of $p$-mutants enjoying the property that the first $K$ elements thereof constitute a covariance-minimizing design. That is, if we denote by $D_{K}^{*}(p)$ the design made of the first $K$ sequences in the list, then

$$
\tau\left(D_{K}^{*}(p)\right) \leq \tau(D), \quad \text { for all } D \in \mathscr{D}_{K}(p), K=1,2, \ldots
$$

We refer to this order as the covariance-minimizing order.

\subsection{Choosing the final design}

Simulations indicate that when $D$ is a covariance-minimizing design in $\mathscr{D}_{K}(p)$, its expected fitness $E\left[F_{D}\right]$ is very close to the approximate upper bound $E\left[U_{K}(p)\right]$ in (16); see Fig. 2. Thus, we may treat $E\left[U_{K}(p)\right]$ as an approximation for $E\left[F_{D}\right]$. Based on this approximation, we now propose a heuristic to find a solution $D$ to the general single-period design problem (12).

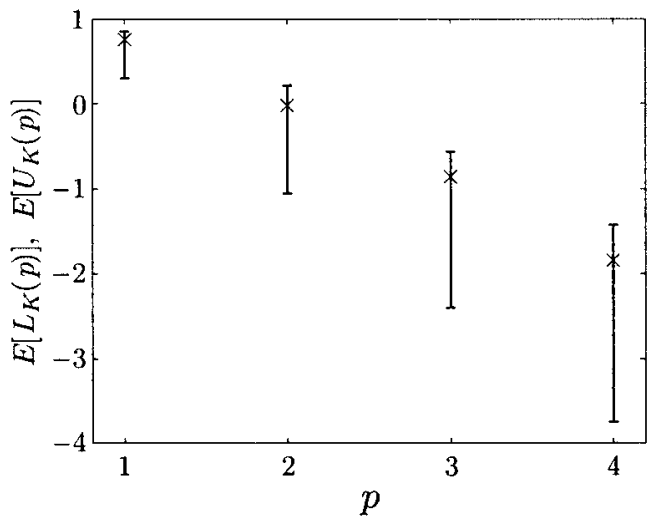

FIG. 2. Simulated values of $E\left[F_{D}\right]$ (" $\times$ ” symbols) for covariance-minimizing designs $D \in \mathscr{D}_{300}$ ( $p$ ) (that is, optimal solutions of (42)), for various values of $p$. The bars indicate the approximate upper and lower bounds $E\left[U_{300}(p)\right]$ and $E\left[L_{300}(p)\right]$ in (16) and (18). Landscape parameters are as estimated in (11), and $n=36$. 

i.e.,

Given $K$ and the landscape parameters $m, \sigma_{\mathrm{S}}^{2}, \sigma_{\mathrm{R}}^{2}$, and $\sigma_{\mathrm{N}}^{2}$, let $p_{(j)}$ be the $j$ th best argument of $E\left[U_{K}(p)\right]$,

$$
\begin{aligned}
& p_{(1)}=\underset{p}{\arg \max }\left\{E\left[U_{K}(p)\right]: p=1,2, \ldots\right\}, \\
& p_{(2)}=\underset{p}{\arg \max }\left\{E\left[U_{K}(p)\right]: p \neq p_{(1)}, \quad p=1,2, \ldots\right\}, \\
& p_{(3)}=\underset{p}{\arg \max }\left\{E\left[U_{K}(p)\right]: p \neq p_{(1)}, p_{(2)}, \quad p=1,2, \ldots\right\},
\end{aligned}
$$

First include in $D$ as many as possible (i.e., without exceeding $K$ ) sequences from $\mathscr{S}_{p_{(1)}}$, in the covarianceminimizing order described in Section 3.3. If $K>\left|\mathscr{S}_{p_{(1)}}\right|$, repeat this process with sequences from $\mathscr{S}_{p_{(2)}}$ and continue this way until $K$ is reached.

To facilitate this process, we think of $E\left[U_{K}(p)\right]$ (see Equation (16)) as a continuous function of $p$. This function depends of course on the value of $K$ and on the values of the landscape parameters; see Fig. 3. This function is concave by (16) and is monotonically increasing on the entire half-line $[0, \infty)$ only when $m \geq 0$, which is never the case. Elementary calculus gives then that the maximum of $E\left[U_{K}(p)\right]$ is attained at

$$
p^{*}=\frac{\sigma_{\mathrm{S}}^{2}+\sigma_{\mathrm{R}}^{2}}{4 m^{2}}\left(b_{K}+\frac{\gamma}{a_{K}}\right)^{2}-\frac{\sigma_{\mathrm{N}}^{2}}{\sigma_{\mathrm{S}}^{2}+\sigma_{\mathrm{R}}^{2}} .
$$

Thus, if $p^{*} \leq 1$, the function $E\left[U_{K}(\cdot)\right]$ is monotonically decreasing on $\{1,2, \ldots\}$, in which case $p_{(j)}=j$. Otherwise, we need to check the value of $E\left[U_{K}(\cdot)\right]$ at the two "integer neighbors" of $p^{*}$ to find $p_{(1)}$, and then continue checking neighbors in the obvious way to find $p_{(2)}, p_{(3)}$, etc. The number of $p$-mutants is $\left|\mathscr{S}_{p}\right|=\left(\begin{array}{l}n \\ p\end{array}\right) 19^{p}$ and grows very rapidly with $p$ even for small values of $n$; therefore, we expect that in almost all cases only one or two types of mutants will be needed.

As the drift becomes weaker (i.e., when the negative parameter $m$ gets closer to zero), the value of $p^{*}$ increases. Thus, our model predicts that drug designers are better off screening variants with multiple mutations in a weak-drift parameter regime. The intuition behind this observation is that the fitness variance of a $p$-mutant is increasing in $p$, whereas its mean fitness is linearly decreasing in $p$, with slope $m$ (see (14)); neglecting covariance effects, when $m$ is close to zero, the decrease in mean fitness due to higher $p$ is not significant, but the higher variance results in a higher mean for the maximum fitness among a collection of variants-a well-known result in extreme value theory.

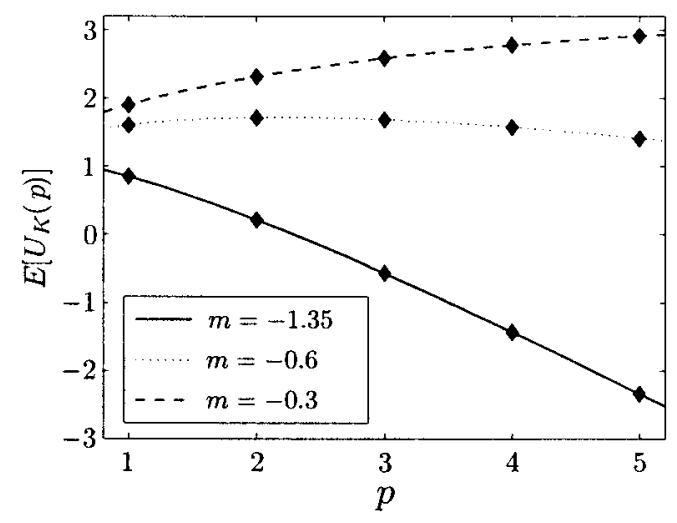

FIG. 3. The approximation $E\left[U_{K}(p)\right]$ as a continuous function of $p$, when $K=300$ and for three values of the drift $m$. The values of $\sigma_{\mathrm{S}}^{2}, \sigma_{\mathrm{R}}^{2}$, and $\sigma_{\mathrm{N}}^{2}$ are as estimated in (11). 


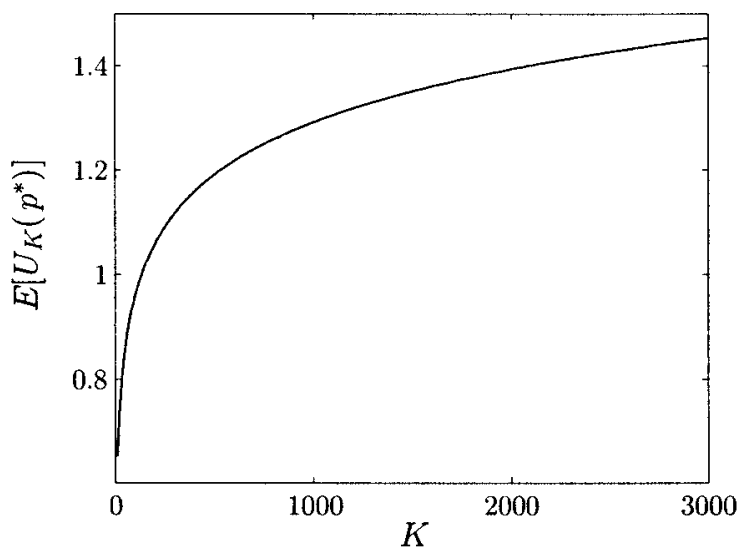

FIG. 4. $E\left[U_{K}\left(p^{*}\right)\right]$ as a function of $K$. Landscape parameters are as estimated in (11).

The value of $p^{*}$ also increases with the sum $\sigma_{\mathrm{S}}^{2}+\sigma_{\mathrm{R}}^{2}$. Indeed, increasing either the site variance $\sigma_{\mathrm{S}}^{2}$ or the residue variance $\sigma_{\mathrm{R}}^{2}$ increases the fitness variance (without affecting the mean) of the various variants, making variants with more mutation more likely to have extremes. Equation (23) also implies that $p^{*}$ is a decreasing function of the nonadditivity variance, $\sigma_{\mathrm{N}}^{2}$.

To study the effect of the landscape parameters on the expected fitness achieved with the optimal $p$, we note that the function $E\left[U_{K}(\cdot)\right]$ in (16) increases uniformly when either of the four landscape parameters increases. Thus, the value of $\max _{p} E\left[U_{K}(p)\right]$ also increases when either $m, \sigma_{\mathrm{S}}^{2}, \sigma_{\mathrm{R}}^{2}$, or $\sigma_{\mathrm{N}}^{2}$ increases. Moreover, the expected maximal fitness depends on $\sigma_{\mathrm{S}}^{2}$ and $\sigma_{\mathrm{R}}^{2}$ only through their sum.

Up to this point, we have treated the capacity $K$ as exogenous and fixed, although capacity is endogenous in practice. The quantity $b_{K}+\gamma / a_{K}$ is increasing in $K$, so for the same reasons as those mentioned in the previous paragraph, the value of $\max _{p} E\left[U_{K}(p)\right]$ increases in $K$. The intuition behind this is obvious: more resources mean a better outcome. To see roughly how fast $\max _{p} E\left[U_{K}(p)\right]$ grows with $K$, we substitute $p^{*}$ from (23) into $E\left[U_{K}(p)\right]$ from (16); doing so provides only an approximation, since $p^{*}$ may be below 1 (or even negative), and since as $K$ grows, the number of mutant classes of size $K$ or more decreases. Nevertheless, we get in this case

$$
E\left[U_{K}\left(p^{*}\right)\right]=-\frac{\sigma_{\mathrm{S}}^{2}+\sigma_{\mathrm{R}}^{2}}{4 m}\left(b_{K}+\frac{\gamma}{a_{K}}\right)^{2}-\frac{m \sigma_{\mathrm{N}}^{2}}{\sigma_{\mathrm{S}}^{2}+\sigma_{\mathrm{R}}^{2}}=O(\log K) ;
$$

that is, $\max _{p} E\left[U_{K}(p)\right]$ grows roughly logarithmically with $K$. Figure 4 shows $E\left[U_{K}\left(p^{*}\right)\right]$ as a function of $K$, when the landscape parameters are the estimated ones from (11). The resulting graph is concave, reflecting a strong diminishing returns to scale with respect to $K$.

\section{THE TWO-PERIOD DESIGN PROBLEM}

Suppose now that we have enough time to afford two rounds of production and screening; this is a realistic situation, as noted by Fox et al. (2003) and Wu et al. (1998). The order of events in this scenario is as follows:

1. Choose a first-period design $D_{1} \subseteq \mathscr{S}$.

2. Screen the variants in $D_{1}$, and observe their fitness values $\mathbf{F}_{1}=\left(F_{s}, s \in D_{1}\right)$.

3. Using the conditional distribution formulas (10), update the fitness distribution of the remaining variants, and based on the updated distribution, choose a second-period design, $D_{2} \subseteq \mathscr{S}$.

4. Screen the variants in $D_{2}$ and keep the variant with the highest fitness found in the entire process. 
Our goal is, therefore, to find a design $D_{1}$ and a rule that assigns to each observed $\mathbf{F}_{1}$ a design $D_{2}\left(\mathbf{F}_{1}\right)$ that will jointly maximize the expected fitness of the best variant found in the process; that is, letting $K_{1}=\left|D_{1}\right|$ and $K_{2}=\left|D_{2}\right|$, we solve

$$
\underset{D_{1}, D_{2}\left(\mathbf{F}_{1}\right)}{\operatorname{maximize}} E\left[\max _{s \in D_{1} \cup D_{2}} F_{s}\right] \quad \text { s.t. } \quad K_{1}+K_{2} \leq K .
$$

The constraint reflects the fact that the dominant factor in the production and screening cost of variants is the variable cost. Just as in the single-period case, this constraint clearly holds with equality in the optimal solution.

We study the second-period strategy in Section 4.1, and address the first-period strategy in Section 4.2; a simulation study of the two-period design problem is presented in Section 4.3.

\subsection{Second-period strategy}

Suppose we are in the second period, so we have already observed $\mathbf{F}_{1}$ and have updated the fitness distribution for the variants outside of $D_{1}$ according to (10). Since the updated mean values depend on the observed $\mathbf{F}_{1}$, it is in general no longer true that the fitness values of all sequences in $\mathscr{S}_{p}$ have the same marginal distribution, and none of our previous results carry over.

However, a reasonable strategy here would be to order the sequences outside of $D_{1}$ in decreasing order of expected fitness and let $D_{2}$ consist of the first $K_{2}$ sequences in this order. To do this, we first compute the $19 n$ values $E\left[f_{i}(r) \mid \mathbf{F}_{1}\right], i=1, \ldots, n, r \neq \hat{r}_{i}$ (see Section 2.2 for the definition of $f_{i}(r)$ ). By the additivity of our fitness model and of the expectation operator, we have that

$$
E\left[F_{s} \mid \mathbf{F}_{1}\right]=\sum_{i: r \neq \hat{r}_{i}} E\left[f_{i}(r) \mid \mathbf{F}_{1}\right], \quad s \notin D_{1} .
$$

Since choosing not to mutate at site $i$ is equivalent to adding a zero to the above sum, we define

$$
V_{i}=\left\{E\left[f_{i}(r) \mid \mathbf{F}_{1}\right]: r \neq \hat{r}_{i}\right\} \cup\{0\}, \quad i=1, \ldots, n,
$$

and then face the following combinatorial optimization problem: given the $n$ sets $V_{1}, \ldots, V_{n}$, consider the set of all sums $S=\left\{v_{1}+\ldots+v_{n}: v_{i} \in V_{i}\right\}$; what are the $K_{2}$ largest elements of $S$ ? In this subsection we present a heuristic algorithm for this problem and derive bounds to study its performance in the special case where $D_{1}$ is $\mathscr{S}_{1}$, the set of all 1-mutants.

As shown by Johnson and Mizoguchi (1978), the problem of finding the largest $K$ elements of $S$ is NPhard, so it is highly unlikely to have an efficient complete solution. Still, we devised a heuristic algorithm to tackle it, which we illustrate now by an example. For ease of exposition, we let $\left|V_{i}\right|=3$ and $n=4$ and use values that are not typical. Let $V=\left(v_{r i}\right)$ be the matrix that has the elements of $V_{i}$ in its $i$ th column, sorted from largest to smallest. In our example,

$$
V=\left(\begin{array}{cccc}
15 & 2 & 13 & 30 \\
8 & 0 & 10 & 7 \\
-4 & -3 & 9 & -6
\end{array}\right)
$$

We start with the best sum, $15+2+13+30=60$; this sum can be thought of as the only sum that can be created from the "table"

$$
\begin{array}{llll}
15 & 2 & 13 & 30 .
\end{array}
$$

Among the entries of $V$ not used thus far, the one that is the closest to the top entry in its column is $v_{22}=0$ (this is so since $2-0=2$ is smaller than $15-8=7,13-10=3$, and $30-7=23$ ). We insert this entry into the table, which is now

$$
\begin{array}{llll}
15 & 2 & 13 & 30 \\
& 0 & &
\end{array} .
$$


Now we have $1 \cdot 2 \cdot 1 \cdot 1=2$ possible sums that can be created from this table: the number 60 we had before, and now also $15+0+13+30=58$. We start the next iteration: the unused entry of $V$ that is closest to the one at the top of its column is $v_{23}=10$, so we insert it into the table, which now has the form

$$
\begin{array}{llll}
15 & 2 & 13 & 30 \\
& 0 & 10 &
\end{array}
$$

Now we can generate $1 \cdot 2 \cdot 2 \cdot 1=4$ sums, the two new of which are $15+2+10+30=57$ and $15+0+10+30=55$. The next entry to insert is $v_{33}=9$, which changes the table to

$$
\begin{array}{cccc}
15 & 2 & 13 & 30 \\
& 0 & 10 & \\
& & 9 &
\end{array}
$$

and raises the number of possible sums to 6 . We continue in this fashion until the number of sums reaches $K_{2}$ and then stop. We may improve this algorithm by running it until we obtain $K_{2}^{\prime}$ sums, for some $K_{2}^{\prime}>K_{2}$, then sort these sums, and choose the top $K_{2}$ of them. Of course, when we apply this algorithm to our problem, we ignore the sums that correspond to sequences that were already screened in $D_{1}$.

This algorithm seems to perform well when run on input generated from simulated fitness landscapes. We ran it on a computer over a small-sized problem $\left(n=8,\left|V_{i}\right|=16, K_{2}=200\right.$ and $\left.K_{2}^{\prime}=5,000\right)$; within less than two seconds, it managed to find the top 174 out of the "true" 200 best sums, where the latter sums were found by exhaustively computing all $4.29 \cdot 10^{9}$ possible sums, a process that took more than an hour.

To assess the effectiveness of this second-period strategy, we study the important special case in which $D_{1}=\mathscr{S}_{1}$, i.e., the case in which we screen in the first period all 1-mutants. Choosing this $D_{1}$ is both tractable and realistic-it was used by Wu et al. (1998) in the actual design of the drug Vitaxin. The remainder of this subsection is devoted to showing that under this choice of $D_{1}$ and under an additional condition on the choice of $D_{2}$, we can find a uniform upper bound on $E\left[F_{D_{2}}\right]$, and that the results of our selection algorithm are sufficiently close to this bound for small values of $p$.

When $D_{1}=\mathscr{S}_{1}$, the mean vector $\mu_{1}$ and the covariance matrix $\Sigma_{11}$ from (9) are, respectively, the constant $19 n$-vector $(m, \ldots, m)^{\mathrm{T}}$ and the $19 n \times 19 n$ matrix having the block form

$$
\boldsymbol{\Sigma}_{11}=\left(\begin{array}{cccc}
\mathbf{C} & \mathbf{0} & \ldots & \mathbf{0} \\
\mathbf{0} & \mathbf{C} & \cdots & \mathbf{0} \\
& \ldots & & \\
\mathbf{0} & \mathbf{0} & \cdots & \mathbf{C}
\end{array}\right)
$$

where $\mathbf{C}$ is a $19 \times 19$ matrix having $\sigma_{\mathrm{S}}^{2}+\sigma_{\mathrm{R}}^{2}+\sigma_{\mathrm{N}}^{2}$ on its diagonal and $\sigma_{\mathrm{S}}^{2}$ off of it (see (6)-(8)). It is a matter of verification to see that $\mathbf{C}^{-1}=\left(c_{i j}^{-1}\right)$ is given by

$$
c_{i i}^{-1}=\Delta^{-1}\left(18 \sigma_{\mathrm{S}}^{2}+\sigma_{\mathrm{R}}^{2}+\sigma_{\mathrm{N}}^{2}\right) \quad \text { and } \quad c_{i j}^{-1}=-\Delta^{-1} \sigma_{\mathrm{S}}^{2}, \quad i \neq j,
$$

where

$$
\Delta=19 \sigma_{\mathrm{S}}^{2}\left(\sigma_{\mathrm{R}}^{2}+\sigma_{\mathrm{N}}^{2}\right)+\left(\sigma_{\mathrm{R}}^{2}+\sigma_{\mathrm{N}}^{2}\right)^{2} .
$$

Then $\boldsymbol{\Sigma}_{11}^{-1}$ has the same block form as $\boldsymbol{\Sigma}_{11}$ in (26), but with $\mathbf{C}^{-1}$ replacing $\mathbf{C}$.

To simplify notation, we denote by $F_{i, r}$ the fitness of the sequence in $\mathscr{S}_{1}$ that has residue $r \neq \hat{r}_{i}$ at site $i$ and also define

$$
\bar{F}_{i}=\frac{1}{19} \sum_{r \neq \hat{r}} F_{i, r} .
$$


After tedious matrix multiplications and cancellations, we get from the above that for two sequences $s$, $s^{\prime} \notin D_{1}$,

$$
\begin{aligned}
E\left[F_{s} \mid \mathbf{F}_{1}\right] & =\Delta^{-1} m \sigma_{\mathrm{N}}^{2}\left(\sigma_{\mathrm{R}}^{2}+\sigma_{\mathrm{N}}^{2}\right) d\left(s, s^{\prime}\right)+\sum_{i: r \neq \hat{r}_{i}}\left[\Delta^{-1} 19 \sigma_{\mathrm{S}}^{2} \sigma_{\mathrm{N}}^{2} \bar{F}_{i}+\frac{\sigma_{\mathrm{R}}^{2}}{\sigma_{\mathrm{R}}^{2}+\sigma_{\mathrm{N}}^{2}} F_{i, r}\right], \\
\operatorname{Var}\left(F_{s} \mid \mathbf{F}_{1}\right) & =\Delta^{-1} d\left(s, s^{\prime}\right)\left(\sigma_{\mathrm{S}}^{2} \sigma_{\mathrm{N}}^{4}+19 \sigma_{\mathrm{S}}^{2} \sigma_{\mathrm{R}}^{2} \sigma_{\mathrm{N}}^{2}+\sigma^{4} \sigma_{\mathrm{N}}^{2}+\sigma_{\mathrm{R}}^{2} \sigma_{\mathrm{N}}^{4}\right)+\sigma_{\mathrm{N}}^{2}, \\
\operatorname{Cov}\left(F_{s}, F_{s^{\prime}} \mid \mathbf{F}_{1}\right) & =\Delta^{-1} \sigma_{\mathrm{S}}^{2} \sigma_{\mathrm{N}}^{4}\left|M_{1}\left(s, s^{\prime}\right)\right|+\Delta^{-1}\left(19 \sigma_{\mathrm{S}}^{2} \sigma_{\mathrm{R}}^{2} \sigma_{\mathrm{N}}^{2}+\sigma_{\mathrm{R}}^{2} \sigma_{\mathrm{N}}^{4}+\sigma^{4} \sigma_{\mathrm{N}}^{2}\right)\left|M_{2}\left(s, s^{\prime}\right)\right| .
\end{aligned}
$$

Note that neither the variance nor the covariance values depend on the observed $\mathbf{F}_{1}$, and that the conditional covariance is always positive. We also compute for each site $i$ the quantity

$$
u_{i}=\max \left\{0, \max _{r \neq \hat{r}_{i}} E\left[f_{i}(r) \mid \mathbf{F}_{1}\right]\right\}, \quad i=1, \ldots, n \text {. }
$$

Recalling that choosing not to mutate at site $i$ is equivalent to adding zero to the sum in (25), we see that $u_{i}$ is the highest contribution to the expected fitness that can be obtained from site $i$.

Suppose we are willing to consider in $D_{2}$ only sequences having $\bar{p}$ or fewer mutations, for some fixed $\bar{p}$, i.e.,

$$
D_{2} \subseteq \mathscr{S}_{2} \cup \mathscr{S}_{3} \cup \cdots \cup \mathscr{S}_{\bar{p}}
$$

We are not allowing 1-mutants, as they were all screened in the first period. It follows from (27) that if $s \in D_{2}$, then

$$
\operatorname{Var}\left(F_{S} \mid \mathbf{F}_{1}\right) \leq \bar{\sigma}^{2} \triangleq \Delta^{-1} \bar{p}\left(\sigma_{\mathrm{S}}^{2} \sigma_{\mathrm{N}}^{4}+19 \sigma_{\mathrm{S}}^{2} \sigma_{\mathrm{R}}^{2} \sigma_{\mathrm{N}}^{2}+\sigma_{\mathrm{R}}^{4} \sigma_{\mathrm{N}}^{2}+\sigma_{\mathrm{R}}^{2} \sigma_{\mathrm{N}}^{4}\right)+\sigma_{\mathrm{N}}^{2}
$$

We shall use this bound $\bar{\sigma}^{2}$ momentarily.

Let $D_{2}$ be a design of size $K_{2}$ as in (30), and let $\mathbf{F}_{2}=\left(F_{s}, s \in D_{2}\right)$. Now define $\bar{\mu}$ to be the sum of the $\bar{p}$ highest $u_{i}$ from (29); by this definition, $\bar{\mu}$ is an upper bound on the expected fitness $E\left[F_{s} \mid \mathbf{F}_{1}\right]$ of a sequence $s \in D_{2}$. We let $\mathbf{G}=\left(G_{i}\right)$ be a multivariate normal vector having the same covariance matrix as that of $\mathbf{F}_{2}$ given $\mathbf{F}_{1}$, but whose mean vector is the constant $K_{2}$-vector $(\bar{\mu}, \ldots, \bar{\mu})^{\mathrm{T}}$ :

$$
E\left[G_{i}\right]=\bar{\mu}, \quad \operatorname{Cov}(\mathbf{G})=\operatorname{Cov}\left(\mathbf{F}_{2} \mid \mathbf{F}_{1}\right) .
$$

A simple pathwise argument yields

$$
E\left[\max \mathbf{F}_{2} \mid \mathbf{F}_{1}\right] \leq E[\max \mathbf{G}],
$$

where $\max \mathbf{X}$ denotes the maximal element in the vector $\mathbf{X}$. Now let $\mathbf{H}=\left(H_{i}\right)$ be a multivariate normal vector having the same mean vector as $\mathbf{G}$, but whose covariance matrix is changed so that its diagonal entries are all equal to $\bar{\sigma}^{2}$ in (31), and its off-diagonal entries are all zero; i.e.,

$$
E\left[H_{i}\right]=\bar{\mu}, \quad \operatorname{Var}\left(H_{i}\right)=\bar{\sigma}^{2}, \quad \text { and } \operatorname{Cov}\left(H_{i}, H_{j}\right)=0, \quad i \neq j .
$$

From the definition of $\bar{\sigma}^{2}$, we have that $\operatorname{Var}\left(G_{i}\right) \leq \operatorname{Var}\left(H_{i}\right)$ for all $i$, and since all the elements of $\mathbf{G}$ and $\mathbf{H}$ have the same mean (namely, $\bar{\mu}$ ), we get

$$
E\left[G_{i}^{2}\right] \leq E\left[H_{i}^{2}\right] \quad \text { for all } i
$$

In addition, the conditional covariance $\operatorname{Cov}\left(F_{s}, F_{s^{\prime}} \mid \mathbf{F}_{1}\right)$ is always nonnegative by (28), and so

$$
\operatorname{Cov}\left(G_{i}, G_{j}\right) \geq \operatorname{Cov}\left(H_{i}, H_{j}\right), \quad i \neq j,
$$



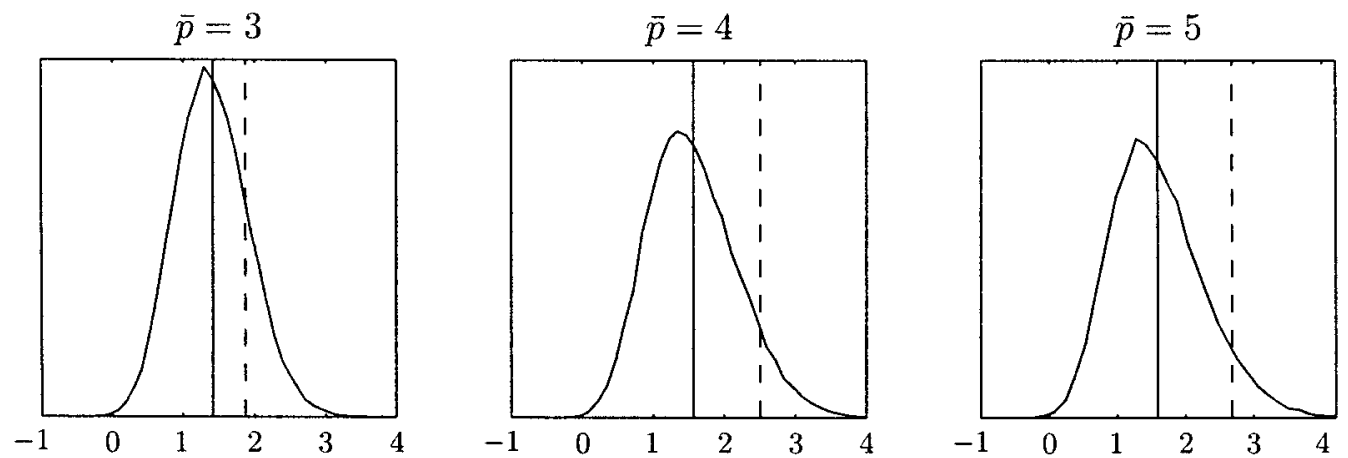

FIG. 5. The distribution and the mean of $F_{D}$ (solid curve and line) when the number of mutations per sequence is at most $\bar{p}$, for various values of $\bar{p}$. The dashed line is the bound on expected fitness from equation (34). Parameters are $n=30, K_{1}=19 n=570$, and $K_{2}=300$; landscape parameters are as estimated in (11).

and using again the equality in means we have

$$
E\left[G_{i} G_{j}\right] \geq E\left[H_{i} H_{j}\right], \quad i \neq j .
$$

Combining (32) and (33), we get

$$
E\left(G_{i}-G_{j}\right)^{2} \leq E\left(H_{i}-H_{j}\right)^{2}, \quad \text { for all } i, j .
$$

It follows now from the Sudakov-Fernique inequality (see Appendix G) that $E[\max \mathbf{G}] \leq E[\max \mathbf{H}]$. The elements of $\mathbf{H}$ are independent and identically distributed normal random variables, so we can again use extreme value theory to approximate their maximum and get from all of the above

$$
E\left[\max \mathbf{F}_{2} \mid \mathbf{F}_{1}\right] \leq E[\max \mathbf{H}] \approx \bar{\mu}+\bar{\sigma}\left(b_{K_{2}}+\frac{\gamma}{a_{K_{2}}}\right) .
$$

Until now, we treated $\mathbf{F}_{1}$ as fixed, and conditioned everything on its value. Note that of the two quantities $\bar{\sigma}$ and $\bar{\mu}$, only $\bar{\mu}$ depends on $\mathbf{F}_{1}$. Thus, when we average the last inequality over all realizations of $\mathbf{F}_{1}$ ( $D_{1}$ is still the set of all 1-mutants) we get

$$
E\left[\max \mathbf{F}_{2}\right] \leq E[\bar{\mu}]+\bar{\sigma}\left(b_{K_{2}}+\frac{\gamma}{a_{K_{2}}}\right),
$$

where the value of $E[\bar{\mu}]$ can be evaluated numerically. In this special case, the right side of (34) is a uniform upper bound on the achievable fitness in the second period: if $D_{1}=\mathscr{S}_{1}$ and $D_{2}$ contains only sequences having $\bar{p}$ or fewer mutations, then no matter how cleverly we choose $D_{2}$, its expected fitness will never exceed this bound.

Figure 5 shows the bound in (34) superimposed on the distribution and mean value of $F_{D_{2}}$ as computed from our algorithm via simulation, for various values of $\bar{p}$. While this bound appears too crude (recall the construction of the mean vector of $\mathbf{H}$ ) to be useful in general, it appears to be reasonably tight for small values of $\bar{p}$ (e.g., less than four). We conjecture that the deterioration of the bound's performance for larger values of $\bar{p}$ is due to the bound itself and that our second-period strategy of choosing for $D_{2}$ the top $K_{2}$ variants (in terms of expected fitness) and our algorithm to implement this strategy are rather efficient.

\subsection{First-period strategy}

There is a tradeoff in the two-period problem between "exploration" and "exploitation." The more sequences we screen in the first stage (i.e., the higher $K_{1}$ is), the better is the information we collect about the realization we are facing and the more informed are our decisions in the second stage. On the other 
hand, high $K_{1}$ means fewer sequences screened in the second period (i.e., lower $K_{2}$ ), which of course limits our ability to find the sequences with high fitness. We therefore view the first period as an "exploration" phase, where we focus on collecting information, rather than on obtaining high fitness values.

Even if we have decided about the size $K_{1}$ of $D_{1}$, there remains the question of which sequences to choose for it. In an attempt to answer this question, recall that our fitness model is driven by relatively few (namely, 19n) random variables - the $f_{i}(r)$ - and that on top of these we have the noise terms $\epsilon_{s}$ (see Section 2.2). Knowing the exact values of these random variables would render the design problem trivial. While there is nothing we can do in the first period to reduce the uncertainty of the $\epsilon_{s}$ for $s \in D_{2}$, a careful choice of $D_{1}$ may significantly reduce the uncertainty of the $f_{i}(r)$.

We take as a measure of uncertainty the conditional differential entropy of $\mathbf{f}=\left(f_{i}(r)\right)$ given $\mathbf{F}_{1}$, which we denote by $h\left(\mathbf{f} \mid \mathbf{F}_{1}\right)$; see Cover and Thomas (1991). Let $\boldsymbol{\Sigma}_{1}$ denote the covariance matrix of the random $K_{1}$-vector $\mathbf{F}_{1}$, and let $\boldsymbol{\Sigma}$ denote the covariance matrix of the joint $\left(19 n+K_{1}\right)$-vector $\left(\mathbf{f}, \mathbf{F}_{1}\right)$. Using basic information-theoretic results, we have

$$
\begin{aligned}
h\left(\mathbf{f} \mid \mathbf{F}_{1}\right) & =h\left(\mathbf{f}, \mathbf{F}_{1}\right)-h\left(\mathbf{F}_{1}\right) \\
& =\frac{1}{2} \log \left((2 \pi e)^{19 n+K_{1}} \operatorname{det} \boldsymbol{\Sigma}\right)-\frac{1}{2} \log \left((2 \pi e)^{K_{1}} \operatorname{det} \boldsymbol{\Sigma}_{1}\right) \\
& =\frac{1}{2} \log (2 \pi e)^{19 n}+\frac{1}{2} \log \left(\frac{\operatorname{det} \boldsymbol{\Sigma}}{\operatorname{det} \boldsymbol{\Sigma}_{1}}\right) .
\end{aligned}
$$

Since the first term in (35) is constant and the log function is monotonically increasing, we are looking for a design $D_{1}$ that minimizes the ratio $\operatorname{det} \boldsymbol{\Sigma} / \operatorname{det} \boldsymbol{\Sigma}_{1}$.

Numerical computations seem to indicate that when we restrict ourselves to designs made of only $p$-mutants (i.e., when $D_{1} \in \mathscr{D}_{K_{1}}(p)$ for some fixed $p$ ), the covariance-minimizing designs from Section 3.3 minimize the above-mentioned ratio. Based on this, and arbitrarily starting with lower-order mutants, we propose to order all sequences as follows: first in the order are all 1-mutants, in a covariance minimizing order; next are all 2-mutants, also in a covariance minimizing order, etc. We then choose $D_{1}$ to be the first $K_{1}$ sequences in this order. This way, we collapse the complex problem of choosing $D_{1}$ into the singleparameter problem of choosing $K_{1}$. In Section 4.3, we study the choice of $K_{1}$ under various parameters regimes via simulation.

\subsection{Simulation study}

After finding a second-period strategy and collapsing the first-period decision into choosing only $K_{1}$, we use simulation in this subsection to study the impact of the landscape parameters-the drift $m$, the nonadditivity variance $\sigma_{\mathrm{N}}^{2}$, and the site variance $\sigma_{\mathrm{S}}^{2}$ divided by the residue variance $\sigma_{\mathrm{R}}^{2}$, which we call the clustering ratio - on the expected fitness $E\left[\max _{s \in D_{1} \cup D_{2}} F_{s}\right]$ and the optimal number of variants screened in the first period, $K_{1}$. Because the total number of screenings $K$ and the number of periods ( 1 or 2 ) are essentially endogenous decisions in the biotechnology industry (although not in our model), we also look at the impact of $K$ on the fitness achieved and assess the value of employing a second time period (i.e., comparing the fitness under the best value of $K_{1}$ versus $K_{1}=K$ ).

We start by studying a protein of length $n=30$ and consider three values for the total number of screenings allowed, $K$ :

- $K=10 n=300$; here $K$ is significantly lower than the number of 1-mutants, $\left|\mathscr{S}_{1}\right|=19 n=570$;

- $K=25 n=750$, so capacity is slightly above $\left|\mathscr{S}_{1}\right|$;

- $K=100 n=3,000$; in this case capacity is well above $\left|\mathscr{S}_{1}\right|$.

All calculations in this subsection are based on 50,000 simulated realizations of the system.

Base case. As our base case for the landscape parameters $m, \sigma_{\mathrm{S}}^{2}, \sigma_{\mathrm{R}}^{2}$, and $\sigma_{\mathrm{N}}^{2}$, we take the estimated Vitaxin parameters from (11). Figure 6 shows the value of $E\left[\max _{s \in D_{1} \cup D_{2}} F_{S}\right]$ (as estimated by simulation) as a function of $K_{1}$ for the three values of $K$. All three graphs appear to be unimodal and concave, 

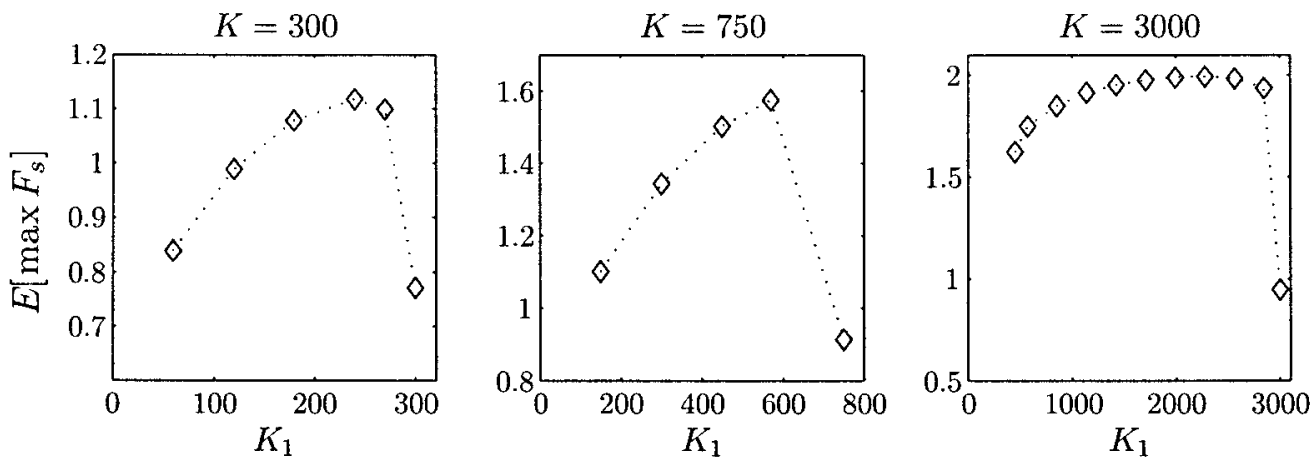

FIG. 6. Mean fitness $E\left[\max _{s \in D_{1} \cup D_{2}} F_{S}\right]$ as a function of $K_{1}$ for three different values of $K$. Landscape parameters are as estimated in (11).

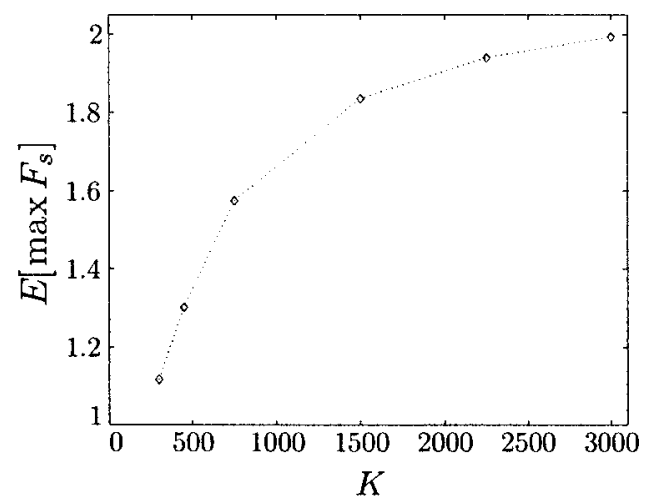

FIG. 7. Best value of $E\left[\max _{s \in D_{1} \cup D_{2}} F_{S}\right]$, achieved with optimal $K_{1}$, for various values of $K$.

consistent with the exploration-exploitation trade-off described above. Also notice the "steep drop" that all three graphs exhibit, leading to their rightmost point; this point corresponds to the choice $K_{1}=K$, where there is no second period, and thus, no learning. This phenomenon demonstrates the importance of exploration: by allocating even very few sequences to the second period, we can significantly improve the outcome.

Obviously, more capacity yields higher fitness: the highest point in the $K=300$ case (about 1.1 ) is lower than that of the $K=750$ case (about 1.6), which in turn is lower than that of the $K=3,000$ case (about 2.0). We can see this phenomenon more clearly in Fig. 7, where the best value of $E\left[\max _{s \in D_{1} \cup D_{2}} F_{S}\right]$, achieved with the optimal $K_{1}$, is plotted for six values of $K$. Just as in the single-period problem, the resulting graph is concave, reflecting a diminishing returns to scale with respect to $K$.

Drift. The drift in the base case is $m=-1.35$. To study the influence of the drift on the mean fitness, we consider a strong-drift regime in which $m=-3$ and a weak-drift regime in which $m=-0.5$; the three other landscape parameters are not changed. As seen in Fig. 8, the strong negative drift causes all fitness values to be very low. An interesting phenomenon in the $K=3,000$ case of Fig. 8 is the "plateau": when the drift is strong, the mean fitness $m d\left(s, s^{\prime}\right)$ of a sequence $s$ drops more rapidly as its distance from the wild type increases, rendering sequences having more than one mutation highly unlikely to possess high fitness. Once all 1-mutants are screened in the first period (i.e., when $K_{1}>\left|\mathscr{S}_{1}\right|=570$ ), the choice of $D_{2}$ in the second period has virtually no effect on the outcome, generating the plateau.

Fitness values soar in the weak-drift regime, where the value of $m$ is changed to -0.5 , as Fig. 9 shows. Notice also how the plateau in the $K=3,000$ case disappears, as a result of the many "attractive" sites that can now be identified in the first period.

Mutational nonadditivity. Next, we study the influence of the nonadditivity variance on expected fitness. In the base case, the noise terms $\epsilon_{s}$ account for a fraction of $\sigma_{\mathrm{N}}^{2} /\left(\sigma_{\mathrm{S}}^{2}+\sigma_{\mathrm{R}}^{2}+\sigma_{\mathrm{N}}^{2}\right)=0.246$ of the variance of 

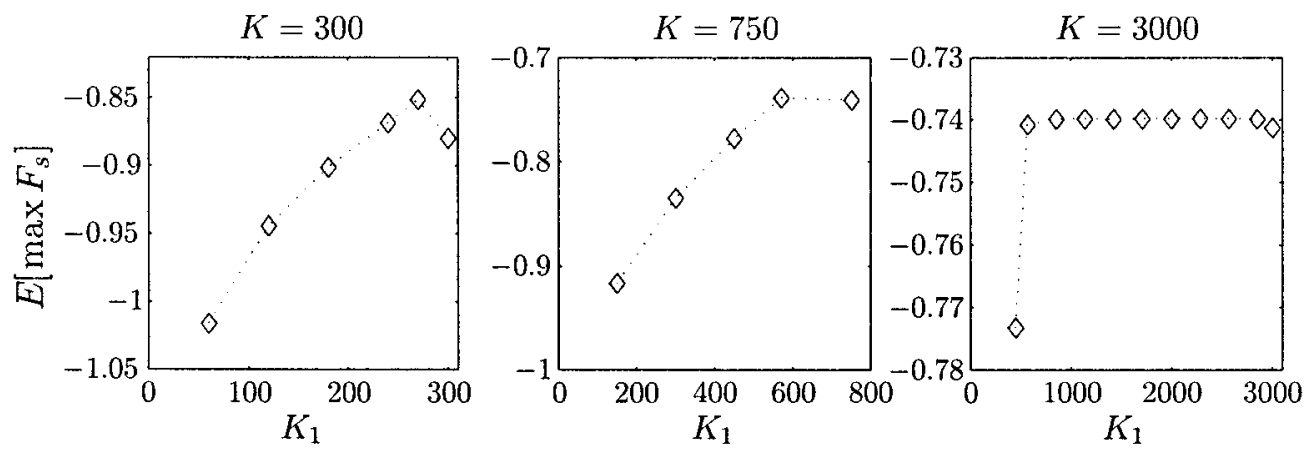

FIG. 8. Mean fitness $E\left[\max _{s \in D_{1} \cup D_{2}} F_{S}\right]$ as a function of $K_{1}$ for three values of $K$ in the strong-drift regime $(m=-3)$.
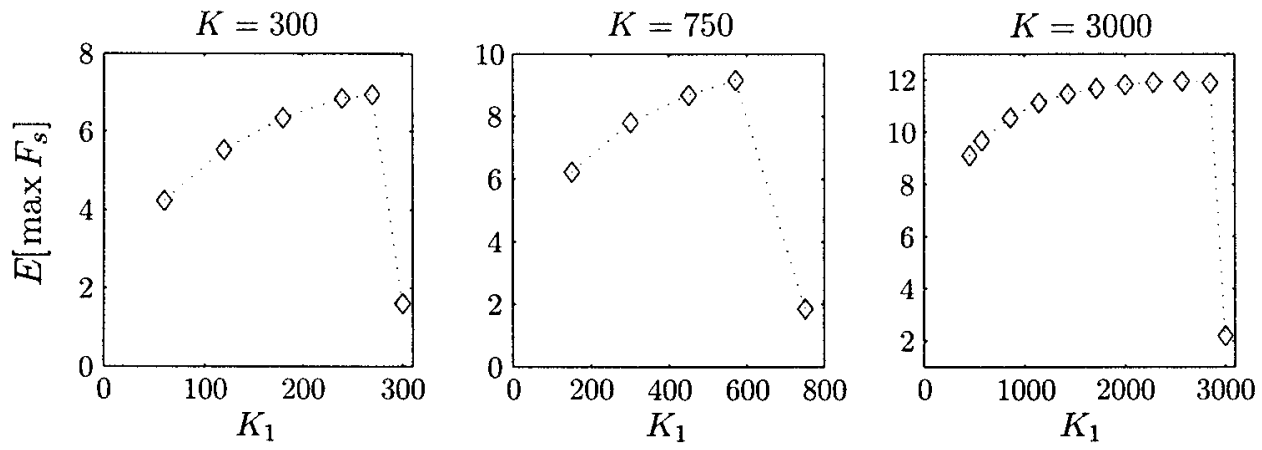

FIG. 9. Mean fitness $E\left[\max _{s \in D_{1} \cup D_{2}} F_{S}\right]$ as a function of $K_{1}$ for three values of $K$ in the weak-drift regime $(m=-0.5)$.
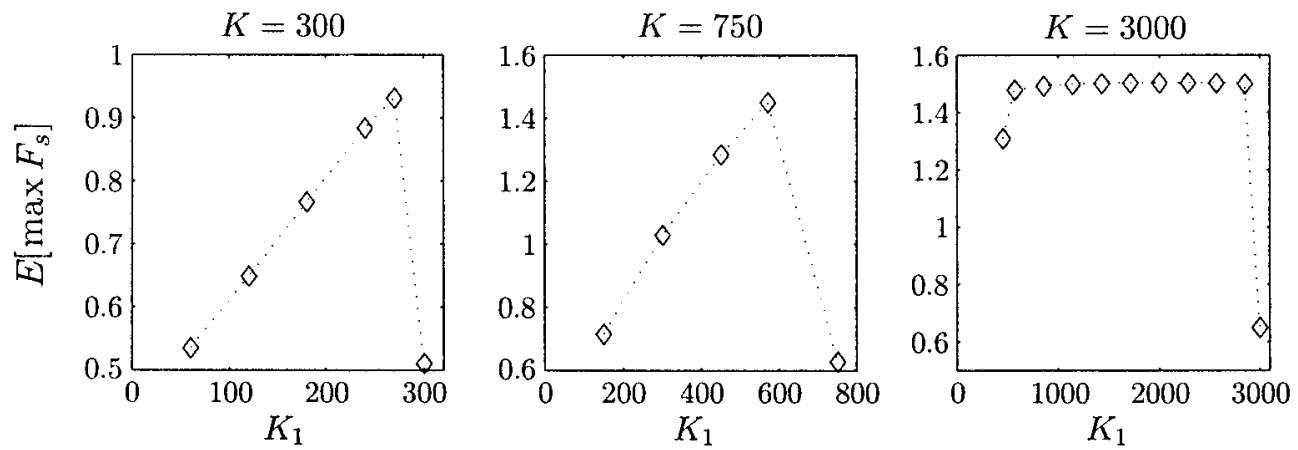

FIG. 10. Mean fitness $E\left[\max _{s \in D_{1} \cup D_{2}} F_{S}\right]$ as a function of $K_{1}$ for three different values of $K$ in the highly-additive regime $\left(\sigma_{\mathrm{N}}^{2}=0.0226\right)$.

a 1-mutant. We consider two other cases here: in the highly additive case, we set $\sigma_{\mathrm{N}}^{2}$ to 0.0226 (and leave all other parameters unchanged), changing this fraction to 0.05 , and in the highly nonadditive case, the value of $\sigma_{\mathrm{N}}^{2}$ is changed to 1.29 , so that the noise terms account for a fraction of 0.75 of the fitness variance of a 1-mutant. Figure 10 shows the relation between $K_{1}$ and $E\left[\max _{s \in D_{1} \cup D_{2}} F_{s}\right]$ in the highly-additive regime. First note how all expected fitness values are uniformly lower than their counterparts in the base case. This is a manifestation of the well-known relation between variance and extreme values (Leadbetter 

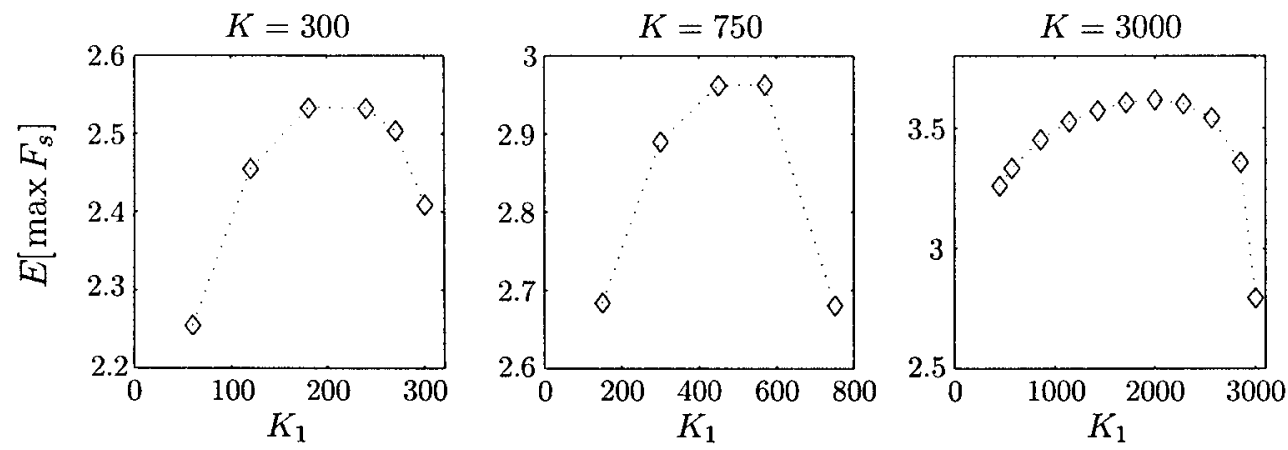

FIG. 11. Mean fitness $E\left[\max _{s \in D_{1} \cup D_{2}} F_{S}\right]$ as a function of $K_{1}$ for three different values of $K$ in the highly nonadditive regime $\left(\sigma_{\mathrm{N}}^{2}=1.29\right)$.
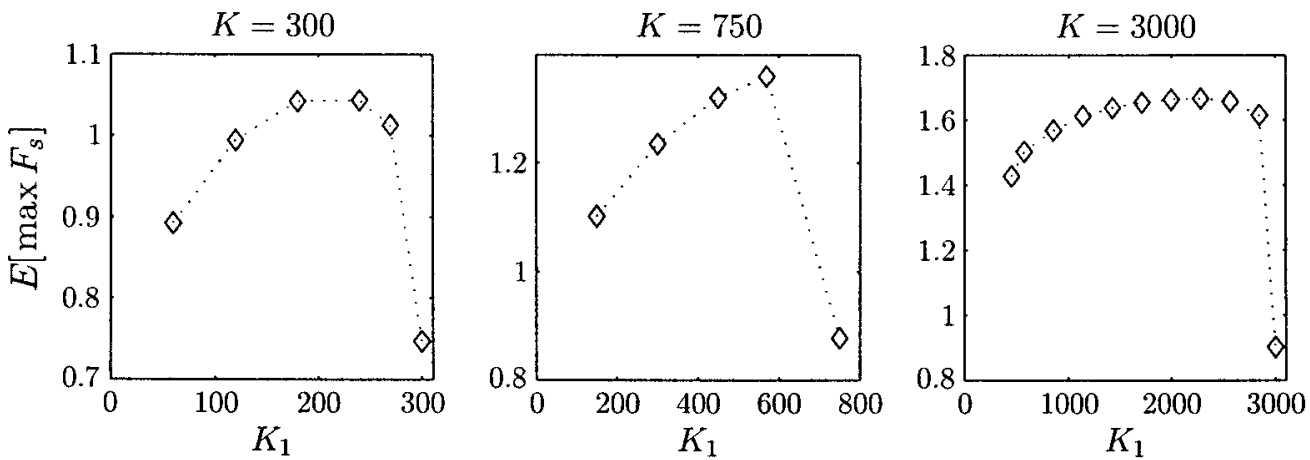

FIG. 12. Mean fitness $E\left[\max _{s \in D_{1} \cup D_{2}} F_{S}\right]$ as a function of $K_{1}$ for three values of $K$ in the high-clustering regime $\left(\sigma_{\mathrm{S}}^{2} / \sigma_{\mathrm{R}}^{2}=2.5\right)$.

et al., 1980). We can also see that a strong drift is not the only cause for the plateau phenomenon. In the $K=3,000$ case of Fig. 10, the plateau is due to information saturation: when there is little nonadditivity, few sequences are needed in the first period to identify the sites and residues that are likely to have a high $f_{i}(r)$. In addition, once these sites and residues are identified, there is not much variability in the second period, so there is also no need for a large $D_{2}$.

Figure 11 depicts the highly nonadditive regime. In addition to the obvious uniform increase in all fitness values, the plateau in the $K=3,000$ case disappears. Indeed, in this noisy environment, many sequences are needed in the first period to collect accurate information on the $f_{i}(r)$, and the exploration-exploitation trade-off is more pronounced. When the parameter $\sigma_{\mathrm{N}}^{2}$ is pushed to a greater extreme (i.e., it accounts for almost all of the fitness variance), the fitness values of the various variants become independent of each other, and no useful information can be obtained in the first period. In such a regime, there is no benefit in a two-period design process, and the designer is better off simply choosing the variants with the highest mean fitness-first as many as possible 1-mutants, then 2-mutants, etc.

Clustering. Holding the sum $\sigma_{\mathrm{S}}^{2}+\sigma_{\mathrm{R}}^{2}$ fixed at 0.43 , we now vary the clustering ratio $\sigma_{\mathrm{S}}^{2} / \sigma_{\mathrm{R}}^{2}$ from the base-case value of $0.25 / 0.18=1.39$ to $\sigma_{\mathrm{S}}^{2} / \sigma_{\mathrm{R}}^{2}=2.5$ in the high-clustering regime, and to $\sigma_{\mathrm{S}}^{2} / \sigma_{\mathrm{R}}^{2}=0.5$ in the low-clustering regime. A comparison of Figs. 6, 12, and 13 reveals that the qualitative results in the base case are the same as in the low-clustering and high-clustering regimes, except that fitness values are higher as the clustering ratio decreases. The reason for this is that reducing the site variance (while increasing the residue variance, but keeping their sum fixed) reduces the covariance between the fitness values of any two variants. As Slepian's inequality shows, lower covariance means higher mean maximal fitness. 

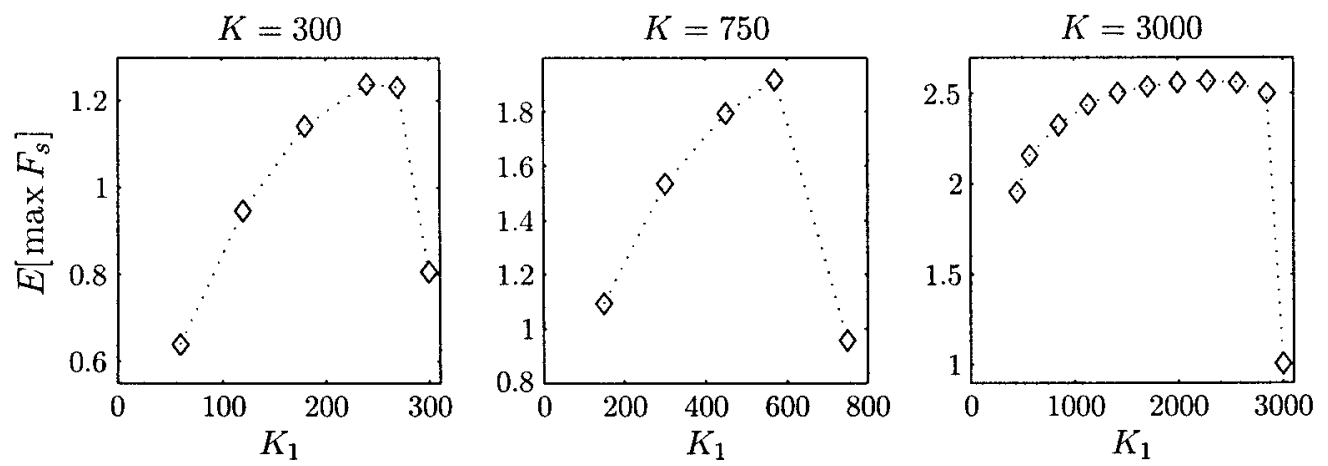

FIG. 13. Mean fitness $E\left[\max _{S \in D_{1} \cup D_{2}} F_{S}\right]$ as a function of $K_{1}$ for three values of $K$ in the low clustering regime $\left(\sigma_{\mathrm{S}}^{2} / \sigma_{\mathrm{R}}^{2}=0.5\right)$.

\section{CONCLUDING REMARKS}

This study has two goals: to develop and validate a new stochastic model for the protein sequence-fitness relation and, in the context of the single-period and two-period design problems, to identify promising strategies and to understand how these strategies are affected by the model's parameter values. With respect to the first goal, while the data of Wu et al. (1998) is insufficient to fully validate the model, the initial statistical results are encouraging. The parameter estimates coincide with long-held views (negative drift, mutational additivity) and with observations from the data (strong clustering). Although published unbiased data sufficient for parameter estimation in our model are rare, additional statistical studies are required both to further validate the sequence-fitness model and to investigate whether different classes of proteins can be segregated into different parameter regimes.

Better data may also allow for model extensions. Some proteins have their active sites grouped in several modules, and these modules sometimes work independently to achieve fitness. For example, antibodies have six complementarity determining regions through which they bind to antigens (Goldsby et al., 2000). We can incorporate modularity into our model by increasing the number of the model parameters and making some of them module specific; for example, we may define $m_{j}$ to be the drift at module $j$. See Perelson and Macken (1994) for a modular version of the $N K$ model. A related approach is to incorporate prior knowledge about the protein's structure, so as to combine traditional rational design with directed evolution (e.g., Altamirano et al., 2000).

Regarding the paper's second goal, mathematical analysis and simulation allow us to gain some understanding of how the model parameters affect the utility and characterization of the protein design strategy. In the single-period design problem, under the base-case parameter values, our analysis suggests to include in the set of screened variants all 1-mutants, then all 2-mutants, etc., until capacity is consumed (and to do so in a covariance-minimizing way). However, for parameter regimes in which either the drift is weak, the mutational nonadditivity is low, the residue variance is high, or the site variance is high, it may be optimal to bypass the 1-mutants and screen only multiple-mutant variants. The mean maximal fitness obtained by the proposed strategy increases when the drift is weak, or when either of the variance terms is high. Also, the mean maximal fitness is concave in the number of screened variants, $K$, and grows roughly as $\log (K)$; however, significant decreasing returns to scale are not experienced until $K$ is large-typically only when it is greater than the total number of 1-mutants.

In the two-period problem, the designer chooses for the first-period design all 1-mutants, then all 2mutants, etc., until the first-period capacity, $K_{1}$, is used up (again, in a covariance-minimizing way). In the second period, the designer uses the fitness values observed in the first period to derive conditional expected fitness values for the unscreened variants and then screens the most promising of these variants in the second period. A key strategic issue in this setting is deciding how many resources to devote to exploration (i.e., first period) versus exploitation (i.e., second period). The two-period analysis shows that the maximal mean fitness is greatly increased by allowing a second period. With two exceptions, it is optimal to have $K_{1} \gg K_{2}$, i.e., most of the screening is devoted to exploration, and relatively few variants 
need to be screened in the exploitation phase. The two exceptions are the strong-drift regime, in which it is difficult to improve over the wild type, and the highly additive regime, in which relatively little exploration is required to identify promising variants for the second period. As in the single-period problem, the mean maximal fitness in the two-period problem increases in the weak-drift and high-nonadditivity regimes, and is concave in the number of screened variants $K$, with severe decreasing returns not incurred until $K$ is larger than the total number of 1-mutants. Reducing the clustering ratio (the site variance $\sigma_{\mathrm{S}}^{2}$ divided by the residue variance $\sigma_{\mathrm{R}}^{2}$ ) while keeping the sum $\sigma_{\mathrm{S}}^{2}+\sigma_{\mathrm{R}}^{2}$ fixed, reduces the fitness covariance between any two variants, and thus increases the mean maximal fitness.

A natural future extension of this analysis is the study of the multiperiod design problem, where more than two screening rounds are performed. This extension may become practically relevant in the coming years, as the throughput rates of production and screening increase and the fixed setup cost and time of each round of screening decrease.

Another direction for future research is to incorporate biochemical expert knowledge of the protein at hand into the model. Drug designers often have a good idea about which residues are likely to be important in determining fitness, which other residues are likely to interact with them, and which changes in them are likely to be conservative or nonconservative. Accommodating this information in the model may significantly improve the design results.

\section{ACKNOWLEDGMENTS}

This research was supported by the Graduate School of Business, Stanford University. We thank Tal Raviv for important help in the analysis of the Integer Program in Appendix F; this work also benefited significantly from conversations with Sridhar Govindarajan and Claes Gustafsson from DNA 2.0 and with Jeffry Watkins from Applied Molecular Evolution.

\section{APPENDIX}

\section{A. Testing for clustering}

In this appendix, we statistically validate the existence of the clustering phenomenon described in Section 2.1. The data set we use is Table 2 of Wu et al. (1998). In this dataset, 23 1-mutant variants were identified having more than threefold improved affinity, and they happened to occur at 14 out of the 73 sites considered. According to a null hypothesis of no clustering, each of these 23 favorable mutations is equally likely to occur at each of the sites, independent of the other mutations. Thus, if we let $N_{i}$ be the number of favorable mutations at site $i$, then the vector $\left(N_{1}, \ldots, N_{73}\right)$ has a multinomial distribution with parameters $(23,1 / 73, \ldots, 1 / 73)$. The number of sites with at least one favorable mutation is $Y=\sum_{i} I_{\left\{X_{i} \geq 1\right\}}$, and its distribution can be easily found by simulation. Based on 100,000 realizations, the probability of the event $\{Y \leq 14\}$ is estimated to be 0.00033 ; thus, we may reject the null hypothesis, and adopt the alternative hypothesis, according to which favorable mutations tend to cluster in relatively few sites.

\section{B. Parameter estimation}

In this appendix, we explain the derivation of the estimated landscape parameters discussed in (11) and describe a goodness-of-fit test we devised for their partial validation.

B.1. The data. Vitaxin is a monoclonal antibody $(\mathrm{mAb})$ that is intended to inhibit the proliferation of new blood vessels-a process called angiogenesis - that occurs around cancerous tumors and also in other abnormal circumstances. It does so by binding to a receptor called $\alpha_{v} \beta_{3}$ that is up-regulated during angiogenesis, so the goal was to find a variant having high binding activity, or using other terminology, that exhibits high affinity to its target. The design efforts focused on the 73 sites constituting the six complementarity-determining regions (CDRs) in the mAb's light and heavy chains. Since one of the heavy chain CDRs is exceptionally long $\left(\operatorname{Trp}^{47}-\mathrm{Gly}^{65}\right)$, it was divided "logically" into two parts, giving rise to a total of seven CDRs. The design process involved two main stages: the creation and screening of 
seven 1-mutant libraries, and the creation and screening of four multiple-site libraries. These stages will be referred to below as stages I and II, respectively.

Stage I. There were three substages within stage I, corresponding to three different screening methods that were used. In the first substage, variants were tested by a technique called capture lift assay, which can tell only whether a variant is better or worse than the wild type. The variants that exhibited better affinity than the wild type were further tested by a second method called ELISA. Unlike the capture lift assay, the ELISA format provides a numerical measure for the affinity of the tested variant, and we refer to it as the ELISA score. Twenty-three variants having more than threefold improved affinity were identified in the ELISA test and are shown in Table 2 of Wu et al. (1998). The affinities of 13 variants out of these 23 were further measured by a third method called surface plasmon resonance. The results of this measure are the association and dissociation rates, $k_{\mathrm{on}}$ and $k_{\mathrm{off}}$, and their (scaled) ratio, $K_{\mathrm{d}}=100 k_{\mathrm{off}} / k_{\mathrm{on}}$, also shown in Table 2 of Wu et al. (1998). Thus, since the seven CDRs considered contain a total of 73 sites, we know at the end of stage I that out of the $19 \times 73=1387$ possible 1-mutants, $1387-23=1364$ variants with known sequences have an ELISA score which is less than three times the ELISA score of the wild type, a number we denote by $\alpha$. We also know the exact $K_{\mathrm{d}}$ score of 13 variants with known sequences and that the remaining $23-13=10$ sequences have ELISA scores above $\alpha$. To convert the unknown ELISA score $\alpha$ to its $K_{\mathrm{d}}$ score equivalent, we take it to be the maximal $K_{\mathrm{d}}$ score among the 13 known $K_{\mathrm{d}}$ scores, namely, 14.3; the idea is that the ELISA and the $K_{\mathrm{d}}$ scores are highly correlated and that 13 observations span a wide enough range so that 10 additional ones, taken roughly from the same distribution, are all likely to fall not far from this range. (Note that high fitness $=$ high affinity $=$ low $K_{\mathrm{d}}$ score.)

Stage II. Based on the sequences of the 23 high-affinity variants found in stage I, four combinatorial libraries, each containing 64 multiple-site mutants, were constructed. The four libraries differ by the mutation chosen in the light chain: it can be either $\mathrm{F}$ at site $32, \mathrm{~N}$ at $92, \mathrm{~L}$ at 96 , or no mutation (wild type $=$ WT). Within each library, we can generate 64 variants according to the choice of mutations in the heavy chain. There are 2 options at site 60 (P and WT), 2 at $97(\mathrm{H}, \mathrm{WT}), 2$ at $101(\mathrm{Y}, \mathrm{WT})$, and 8 at $102(\mathrm{~S}, \mathrm{~T}$, $D, E, M, G, A$, and WT), giving a total of $2 \times 2 \times 2 \times 8=64$ combinations. Although there are in principle 256 variants in total in those libraries, only 242 of them are new; i.e., they are not 1-mutants that were already considered in stage I. The variants from the combinatorial libraries were screened, and 14 variants displaying high affinity above some unreported threshold were identified. These 14 variants, along with their $K_{\mathrm{d}}$ score, are reported in Table 3 of Wu et al. (1998). Thus, in addition to what we knew at the end of stage I, we know at the end of stage II the $K_{\mathrm{d}}$ scores and the sequences of 14 new variants and also know that $242-14=228$ variants with known sequences have some low affinity. In absence of further information, it is reasonable to assume again that the correlation between the $K_{\mathrm{d}}$ score and the score that was used in the screening at this stage is high enough, so that the $K_{\mathrm{d}}$ scores of these latter 228 variants are higher than the maximum $K_{\mathrm{d}}$ score of the former 14 variants; i.e., they are above 1.5.

As a final measure of fitness, we chose $F_{s}=\log _{10}\left(K_{\mathrm{d}}(\hat{s}) / K_{\mathrm{d}}(s)\right)=\log _{10}\left(27.6 / K_{\mathrm{d}}(s)\right)$. This choice indeed guarantees that $F_{\hat{s}}=0$.

B.2. The estimation problem. Based on the above, the estimation problem we are facing can be described as follows. Consider a multivariate normal vector $\mathbf{X}=\left(X_{1}, \ldots, X_{q}\right)^{\mathrm{T}}$, taken from an $N(\boldsymbol{\mu}, \boldsymbol{\Sigma})$ distribution, where $\boldsymbol{\mu}=\boldsymbol{\mu}(\boldsymbol{\theta})$ and $\boldsymbol{\Sigma}=\boldsymbol{\Sigma}(\boldsymbol{\theta})$ are both functions of a more "basic" vector of parameters $\boldsymbol{\theta}=\left(\theta_{1}, \ldots, \theta_{k}\right)$, which we wish to estimate. In our model, $\boldsymbol{\theta}=\left(m, \sigma_{\mathrm{S}}^{2}, \sigma_{\mathrm{R}}^{2}, \sigma_{\mathrm{N}}^{2}\right)$. We observe a single and partially truncated realization of $\mathbf{X}$ : some of the components of the vector, which without loss of generality can be taken to be the first $l$, are fully observed, so we know that $X_{1}=y_{1}, \ldots, X_{l}=y_{l}$; each of the next $p$ components is truncated from above; i.e., we only know that $X_{l+1} \geq a_{l+1}, \ldots, X_{l+p} \geq a_{l+p}$; and each of the remaining $q-l-p$ components is truncated from below, giving $X_{l+p+1} \leq b_{l+p+1}, \ldots, X_{q} \leq b_{q}$. Our data, therefore, can be summarized by the vector $(\mathbf{y}, \mathbf{a}, \mathbf{b})=\left(y_{1}, \ldots, y_{l}, a_{l+1}, \ldots, a_{l+p}, b_{l+p+1}, \ldots, b_{q}\right)$.

Using this notation, we have in our dataset

- $q=1387+242=1,629$;

- $l=13+14=27$;

- $p=10$

- $a_{i}=\log _{10}(27.6 / 14.3)=0.2856, \quad i=28, \ldots, 37$;

- $b_{i}= \begin{cases}0.2856 & i=38, \ldots, 1,401 \\ \log _{10}(27.6 / 1.5)=1.264 & i=1,402, \ldots, 1,629 .\end{cases}$ 
The mean vector $\boldsymbol{\mu}=\boldsymbol{\mu}(m)$ in our case is a 1,629-vector, whose $i$ th element is $d m$, where $d$ is the Hamming distance between the $i$ th sequence and the wild type. The covariance matrix $\Sigma$ is a $1,629 \times$ 1,629 matrix, whose elements can be determined according to (7)-(8).

B.3. The expectation-maximization algorithm. The expectation-maximization (EM) algorithm is an iterative procedure to find maximum likelihood estimates (MLEs) for the parameters of a distribution when the available data is truncated or partially missing. The algorithm was first fully introduced by Dempster et al. (1977), and a comprehensive treatment of many of its variants can be found in McLachlan and Krishnan (1997).

Let $f(\cdot ; \boldsymbol{\theta})$ be the density function of $\mathbf{X}$, so that for $\mathbf{x}=\left(x_{1}, \ldots, x_{q}\right)^{\mathrm{T}}$,

$$
f(\mathbf{x} ; \boldsymbol{\theta})=\frac{1}{(2 \pi)^{q / 2}|\boldsymbol{\Sigma}(\boldsymbol{\theta})|^{1 / 2}} \exp \left\{-\frac{1}{2}(\mathbf{x}-\boldsymbol{\mu}(\boldsymbol{\theta}))^{\mathrm{T}} \boldsymbol{\Sigma}(\boldsymbol{\theta})^{-1}(\mathbf{x}-\boldsymbol{\mu}(\boldsymbol{\theta}))\right\} .
$$

Also let

$$
A=A(\mathbf{a}, \mathbf{b})=\underset{i=l+1}{\stackrel{l+p}{\times}}\left[a_{i}, \infty\right) \underset{i=l+p+1}{\stackrel{q}{\times}}\left(-\infty, b_{i}\right]
$$

so that the conditional density of $\left(X_{l+1}, \ldots, X_{q}\right)$ given the data is

$$
g\left(x_{l+1}^{\prime}, \ldots, x_{q}^{\prime} ; \boldsymbol{\theta}, \mathbf{y}, \mathbf{a}, \mathbf{b}\right)=\frac{f\left(\mathbf{y}, x_{l+1}^{\prime}, \ldots, x_{q}^{\prime} ; \boldsymbol{\theta}\right)}{\int_{A} f\left(\mathbf{y}, u_{l+1}, \ldots, u_{q} ; \boldsymbol{\theta}\right) d u_{l+1} \cdots d u_{q}} .
$$

To write the likelihood of the data $(\mathbf{y}, \mathbf{a}, \mathbf{b})$, we follow both Cox and Oakes (1984) and McLachlan and Krishnan (1997), and integrate the density function $f(\cdot ; \boldsymbol{\theta})$ over the set $A$ across the truncated coordinates; i.e., the likelihood of the parameters given the data $(\mathbf{y}, \mathbf{a}, \mathbf{b})$ is

$$
L(\boldsymbol{\theta} ; \mathbf{y}, \mathbf{a}, \mathbf{b})=\int_{A} f\left(\mathbf{y}, u_{l+1}, \ldots, u_{q} ; \boldsymbol{\theta}\right) d u_{l+1} \cdots d u_{q} .
$$

Our goal is to find an MLE for $\boldsymbol{\theta}$, i.e., to find $\hat{\boldsymbol{\theta}}$ maximizing $L(\boldsymbol{\theta} ; \mathbf{y}, \mathbf{a}, \mathbf{b})$.

Let $L_{c}(\boldsymbol{\theta} ; \mathbf{x})$ be the likelihood function of $\boldsymbol{\theta}$ given the complete data $\mathbf{x}$, i.e., $L_{c}(\boldsymbol{\theta} ; \mathbf{x})=f(\mathbf{x} ; \boldsymbol{\theta})$, and also define the $\log$-likelihood function given the complete data to be $l_{c}(\boldsymbol{\theta} ; \mathbf{x})=\log L_{c}(\boldsymbol{\theta} ; \mathbf{x})$. The EM algorithm starts with assigning arbitrary values to the unknown parameter vector, which we denote by $\boldsymbol{\theta}_{0}$. Each iteration of the algorithm consists of two steps: the expectation (E) step and the maximization (M) step. In the $(j+1)$ st E-step of the EM algorithm, given the current estimate $\boldsymbol{\theta}_{j}$ of the parameters, we need to compute for arbitrary $\boldsymbol{\theta}$ the function

$$
\begin{aligned}
Q\left(\boldsymbol{\theta}, \boldsymbol{\theta}_{j}\right)= & E_{\boldsymbol{\theta}_{j}}\left[l_{c}(\boldsymbol{\theta} ; \mathbf{X}) \mid \mathbf{y}, \mathbf{a}, \mathbf{b}\right] \\
= & \int_{A} l_{c}\left(\boldsymbol{\theta} ; \mathbf{y}, u_{l+1}, \ldots, u_{q}\right) g\left(u_{l+1}, \ldots, u_{q} ; \boldsymbol{\theta} j, \mathbf{y}, \mathbf{a}, \mathbf{b}\right) d u_{l+1} \cdots d u_{q} \\
= & \text { const }-\frac{1}{2} \log |\boldsymbol{\Sigma}(\boldsymbol{\theta})|-\frac{1}{2} \boldsymbol{\mu}(\boldsymbol{\theta})^{\mathrm{T}} \boldsymbol{\Sigma}(\boldsymbol{\theta})^{-1} \boldsymbol{\mu}(\boldsymbol{\theta}) \\
& +\int_{A}\left[(\mathbf{y}, \mathbf{u})^{\mathrm{T}} \boldsymbol{\Sigma}(\boldsymbol{\theta})^{-1} \boldsymbol{\mu}(\boldsymbol{\theta})-\frac{1}{2}(\mathbf{y}, \mathbf{u})^{\mathrm{T}} \boldsymbol{\Sigma}(\boldsymbol{\theta})^{-1}(\mathbf{y}, \mathbf{u})\right] g(\mathbf{u} ; \boldsymbol{\theta} j, \mathbf{y}, \mathbf{a}, \mathbf{b}) d \mathbf{u} .
\end{aligned}
$$

In the $(j+1)$ st M-step, we need to find $\boldsymbol{\theta}$ that maximizes $Q\left(\boldsymbol{\theta}, \boldsymbol{\theta}_{j}\right)$ and have this $\boldsymbol{\theta}$ be the $(j+1)$ st estimate:

$$
\boldsymbol{\theta}_{j+1}=\underset{\theta}{\arg \max } Q\left(\boldsymbol{\theta}, \boldsymbol{\theta}_{j}\right) .
$$

Under very general conditions, the sequence $\left\{\boldsymbol{\theta}_{j}\right\}$ converges to $\hat{\boldsymbol{\theta}}$, the MLE of the data.

In our case, the last expression in (36) involves an integral of a very high dimension, as well as the inverse and the determinant of a very large covariance matrix. Since evaluating $Q$, let alone optimizing 
over it, is a formidable task, we divide our data into two sets. The first set consists of the 1-mutant data from stage I of the design process; this set, which contains the bulk of the data, is relatively tractable analytically, and we use it in Section B.5 to estimate the values of $m, \sigma_{\mathrm{S}}^{2}$, and the sum $\sigma_{\mathrm{R}}^{2}+\sigma_{\mathrm{N}}^{2}$. The second set consists of the multiple-site mutation data from stage II of the design process; this set is small enough to allow numerical evaluation of certain essential quantities, and we use it in Section B.6 to break the sum $\sigma_{\mathrm{R}}^{2}+\sigma_{\mathrm{N}}^{2}$ into its components.

B.4. Maximum likelihood estimation based on full 1-mutants data. In this subsection, we derive closedform expressions for the MLEs of some of the parameters of our model, based on complete (i.e., not truncated) observations of all 19n 1-mutants. This will help us in Section B.5 to estimate the parameters when facing truncated data. In the following, we denote by $k$ the number of possible substitutions at each site (i.e., $k=19$ ).

If we let $F_{i, r}$ be the fitness of the 1-mutant sequence having residue $r$ at site $i$, then (6)-(8) imply that

$$
\begin{aligned}
& E\left(F_{i, r}\right)=m \quad \operatorname{Cov}\left(F_{i, r}, F_{i, r^{\prime}}\right)=\sigma_{\mathrm{S}}^{2}, \quad r \neq r^{\prime}, \\
& \operatorname{Var}\left(F_{i, r}\right)=\sigma_{\mathrm{S}}^{2}+\sigma_{\mathrm{R}}^{2}+\sigma_{\mathrm{N}}^{2} \quad \operatorname{Cov}\left(F_{i, r}, F_{i^{\prime}, r^{\prime}}\right)=0, \quad i \neq i^{\prime} .
\end{aligned}
$$

We can therefore think of the data as $n$ independent realizations $\mathbf{F}_{1}, \ldots, \mathbf{F}_{n}$ of a multivariate normal $k$-vector having mean vector $\mathbf{m}=(m, m, \ldots, m)^{\mathrm{T}}$ and covariance matrix $\mathbf{V}=\left(v_{i j}\right)$ of the form

$$
v_{i i}=\sigma_{\mathrm{S}}^{2}+\sigma_{\mathrm{R}}^{2}+\sigma_{\mathrm{N}}^{2}, \quad v_{i j}=\sigma_{\mathrm{S}}^{2}, \quad i \neq j .
$$

Note that the parameters $\sigma_{\mathrm{R}}^{2}$ and $\sigma_{\mathrm{N}}^{2}$ always appear as a sum in the above and are therefore not estimable separately (this is so because we are dealing with only 1-mutants). Defining $t^{2}=\sigma_{\mathrm{R}}^{2}+\sigma_{\mathrm{N}}^{2}$, we derive below MLEs for the three parameters $m, \sigma_{\mathrm{S}}^{2}$, and $t^{2}$.

The likelihood of the parameters given the data $\mathbf{D}=\left(\mathbf{F}_{1}, \ldots, \mathbf{F}_{n}\right)$ is

$$
L_{c}\left(m, \sigma_{\mathrm{S}}^{2}, t^{2} ; \mathbf{D}\right)=\prod_{i=1}^{n} \frac{1}{(2 \pi)^{k / 2}|\mathbf{V}|^{1 / 2}} \exp \left\{-\frac{1}{2}\left(\mathbf{F}_{i}-\mathbf{m}\right)^{\mathrm{T}} \mathbf{V}^{-1}\left(\mathbf{F}_{i}-\mathbf{m}\right)\right\},
$$

so the log likelihood is, up to an increasing linear transformation,

$$
\begin{aligned}
l_{c}\left(m, \sigma_{\mathrm{S}}^{2}, t^{2} ; \mathbf{D}\right) & =-n \log |\mathbf{V}|-\sum_{i=1}^{n}\left(\mathbf{F}_{i}-\mathbf{m}\right)^{\mathrm{T}} \mathbf{V}^{-1}\left(\mathbf{F}_{i}-\mathbf{m}\right) \\
& =-n \log |\mathbf{V}|-n \mathbf{m}^{\mathrm{T}} \mathbf{V}^{-1} \mathbf{m}-\sum_{i=1}^{n} \mathbf{F}_{i}^{\mathrm{T}} \mathbf{V}^{-1} \mathbf{F}_{i}+2 \sum_{i=1}^{n} \mathbf{F}_{i}^{\mathrm{T}} \mathbf{V}^{-1} \mathbf{m} .
\end{aligned}
$$

It is a matter of verification to see that $\mathbf{V}^{-1}=\left(v_{i j}^{-1}\right)$ is given by

$$
v_{i i}^{-1}=\frac{t^{2}+\sigma_{\mathrm{S}}^{2}(k-1)}{k t^{2} \sigma_{\mathrm{S}}^{2}+t^{4}}, \quad v_{i j}^{-1}=-\frac{\sigma_{\mathrm{S}}^{2}}{k t^{2} \sigma_{\mathrm{S}}^{2}+t^{4}}, \quad i \neq j .
$$

To find $|\mathbf{V}|$, we use the fact that the determinant of a matrix equals the product of its eigenvalues. It is again a matter of verification to see that $\mathbf{V}$ has the eigenvalue $k t^{2}+\sigma_{\mathrm{S}}^{2}$ corresponding to the eigenvector $(1,1, \ldots, 1)^{\mathrm{T}}$, and the eigenvalue $\sigma_{\mathrm{S}}^{2}$ with multiplicity $k-1$ corresponding to the $k-1$ eigenvectors

$$
\left(\begin{array}{c}
-1 \\
1 \\
0 \\
\vdots \\
0
\end{array}\right),\left(\begin{array}{c}
-1 \\
0 \\
1 \\
\vdots \\
0
\end{array}\right), \ldots,\left(\begin{array}{c}
-1 \\
0 \\
0 \\
\vdots \\
1
\end{array}\right) .
$$

Thus, $|\mathbf{V}|=\sigma_{\mathrm{S}}^{2(k-1)}\left(k t^{2}+\sigma_{\mathrm{S}}^{2}\right)$. 
Now we have all the ingredients to write down the log likelihood symbolically as a function of the three parameters $m, \sigma_{\mathrm{S}}^{2}$, and $t^{2}$. By differentiating this function with respect to these parameters, equating to zero, and solving the resulting system of equations, we get the estimates

$$
\hat{m}=\frac{S}{n k}, \quad \hat{\sigma}_{0}^{2}=\frac{Q}{n k(k-1)}-\frac{S^{2}}{n^{2} k^{2}}, \quad \hat{t}^{2}=\frac{P}{n k}-\frac{Q}{n k(k-1)},
$$

where $S, P$, and $Q$ are defined by

$$
S=\sum_{i=1}^{n} \sum_{r=1}^{k} F_{i, r}, \quad P=\sum_{i=1}^{n} \sum_{r=1}^{k} F_{i, r}^{2}, \quad Q=\sum_{i=1}^{n} \sum_{r=1}^{k} \sum_{r^{\prime} \neq r} F_{i, r} F_{i, r^{\prime}}
$$

B.5. EM estimation based on truncated single-point mutations data. The results in Section B.4 put us in a position to combine analytic and numeric methods and estimate $m, \sigma_{\mathrm{S}}^{2}$, and $t^{2}$ given truncated data by the EM algorithm. Let $a=0.2856$ be the "fitness equivalent" of the ELISA score $\alpha$, as described in Section B.1. Then the data of Table 2 in Wu et al. (1998) can be thought of as 73 random 19-vectors $\mathbf{F}_{1}, \ldots, \mathbf{F}_{73}$, out of which

- 59 vectors have all of their coordinates less than $a$;

- five vectors have one coordinate greater than $a$, and the rest less than $a$;

- five vectors have one coordinate with exact known value, and the rest less than $a$;

- three vectors have one coordinate with exact known value, another coordinate which is greater than $a$, and the rest less than $a$;

- one vector has five coordinates with exact known value, two which are greater than $a$, and the rest less than $a$.

Let $\mathbf{D}=\left(\mathbf{F}_{1}, \ldots, \mathbf{F}_{73}\right)$ denote the data. In the $(j+1)$ st E-step of the EM algorithm, given our current estimate $\boldsymbol{\theta}_{j}=\left(m_{j}, \sigma_{\mathrm{S}, j}^{2}, t_{j}^{2}\right)$, we need to compute for arbitrary $\boldsymbol{\theta}=\left(m, \sigma_{\mathrm{S}}^{2}, t^{2}\right)$ the function

$$
\begin{aligned}
Q\left(\boldsymbol{\theta}, \boldsymbol{\theta}_{j}\right)= & E_{\boldsymbol{\theta}_{j}}\left[l_{c}(\boldsymbol{\theta}) \mid \mathbf{D}\right] \\
= & -n \log |\mathbf{V}(\boldsymbol{\theta})|-n \mathbf{m}(\boldsymbol{\theta})^{\mathrm{T}} \mathbf{V}^{-1}(\boldsymbol{\theta}) \mathbf{m}(\boldsymbol{\theta}) \\
& \quad-\sum_{i=1}^{n} E_{\boldsymbol{\theta}_{j}}\left[\mathbf{F}_{i}^{\mathrm{T}} \mathbf{V}^{-1}(\boldsymbol{\theta}) \mathbf{F}_{i} \mid \mathbf{D}\right]+2 \sum_{i=1}^{n} E_{\boldsymbol{\theta}_{j}}\left[\mathbf{F}_{i}^{\mathrm{T}} \mathbf{V}^{-1}(\boldsymbol{\theta}) \mathbf{m}(\boldsymbol{\theta}) \mid \mathbf{D}\right] .
\end{aligned}
$$

From Section B.4, we know that

$$
-n \log |\mathbf{V}(\boldsymbol{\theta})|=-n \log \left(\sigma_{\mathrm{S}}^{2(k-1)}\left(k t^{2}+\sigma_{\mathrm{S}}^{2}\right)\right),
$$

and it is straightforward to show, using (37), that

$$
-n \mathbf{m}(\boldsymbol{\theta})^{\mathrm{T}} \mathbf{V}^{-1}(\boldsymbol{\theta}) \mathbf{m}(\boldsymbol{\theta})=-\frac{n m^{2} k}{k \sigma_{\mathrm{S}}^{2}+t^{2}} .
$$

As for the conditional expectations, we have

$$
E_{\boldsymbol{\theta}_{j}}\left[\mathbf{F}_{i}^{\mathrm{T}} \mathbf{V}^{-1}(\boldsymbol{\theta}) \mathbf{m}(\boldsymbol{\theta}) \mid \mathbf{D}\right]=\frac{m}{k \sigma_{\mathrm{S}}^{2}+t^{2}} \sum_{r=1}^{k} E_{\boldsymbol{\theta}_{j}}\left[F_{i, r} \mid \mathbf{D}\right]
$$

and

$$
E_{\boldsymbol{\theta}_{j}}\left[\mathbf{F}_{i}^{\mathrm{T}} \mathbf{V}^{-1}(\boldsymbol{\theta}) \mathbf{F}_{i} \mid \mathbf{D}\right]=\frac{t^{2}+\sigma_{\mathrm{S}}^{2}(k-1)}{k t^{2} \sigma_{\mathrm{S}}^{2}+t^{4}} \sum_{r=1}^{k} E_{\boldsymbol{\theta}_{j}}\left[F_{i, r}^{2} \mid \mathbf{D}\right]-\frac{\sigma_{\mathrm{S}}^{2}}{k t^{2} \sigma_{\mathrm{S}}^{2}+t^{4}} \sum_{r=1}^{k} \sum_{r^{\prime} \neq r} E_{\boldsymbol{\theta}_{j}}\left[F_{i, r} F_{i, r^{\prime}} \mid \mathbf{D}\right] .
$$


So we have factored $\boldsymbol{\theta}$ out of all expectation operators, and the problem of calculating $Q\left(\boldsymbol{\theta}, \boldsymbol{\theta}_{j}\right)$ from (39) boils down to calculating conditional expectations of the form

$$
E_{\boldsymbol{\theta}_{j}}\left[F_{i, r} \mid \mathbf{D}\right], \quad E_{\boldsymbol{\theta}_{j}}\left[F_{i, r}^{2} \mid \mathbf{D}\right], \quad E_{\boldsymbol{\theta}_{j}}\left[F_{i, r} F_{i, r^{\prime}} \mid \mathbf{D}\right],
$$

which do not involve $\boldsymbol{\theta}$ any more. These expectations are in principle integrals of dimension between 16 and 19 (depending on the type of the vector, of the five types described in the beginning of this subsection), but using the representation (2), we can reduce them to one-dimensional integrals as follows.

Consider, for example, a site $i$ such that the vector $\mathbf{F}_{i}$ is of type 1 according to the above classification; i.e., we only know that all $k=19$ coordinates of this vector are below the value $a$. To evaluate a conditional expectation of the first type in (40), we have

$$
E_{\boldsymbol{\theta}_{j}}\left[F_{i, r} \mid \mathbf{D}\right]=\int_{x_{1}=-\infty}^{a} \cdots \int_{x_{k}=-\infty}^{a} x_{1} f\left(x_{1}, \ldots, x_{k} \mid \boldsymbol{\theta}_{j}\right) d x_{1} \ldots d x_{k},
$$

where $f\left(\cdot \mid \boldsymbol{\theta}_{j}\right)$ is the density function of the $k$-dimensional $N\left(\mathbf{m}\left(\boldsymbol{\theta}_{j}\right), \mathbf{V}\left(\boldsymbol{\theta}_{j}\right)\right)$ distribution, scaled so that its total mass over the region $(-\infty, a]^{k}$ is 1 . If we let $\mathbf{D}_{i}$ denote the event $\left\{F_{i, 1}, \ldots, F_{i, k} \leq a\right\}$, then suppressing the dependence on the parameters $\boldsymbol{\theta}_{j}$ gives

$$
E\left[F_{r, 1} \mid \mathbf{D}_{i}\right]=\frac{E\left[F_{i, 1} ; \mathbf{D}_{i}\right]}{P\left(\mathbf{D}_{i}\right)} .
$$

Using the independence of the building blocks in (2), the denominator of the latter expression can be written as

$$
\begin{aligned}
P\left(\mathbf{D}_{i}\right) & =P\left(\mu_{i}+\hat{f}_{i}(1) \leq a, \ldots, \mu_{i}+\hat{f}_{i}(k) \leq a\right), \\
& =\int_{z_{0}=-\infty}^{\infty} \int_{z_{1}=-\infty}^{a-z_{0}} \cdots \int_{z_{k}=-\infty}^{a-z_{0}} f_{\mu_{i}}\left(z_{0}\right) \prod_{l=1}^{k} f_{\hat{f}_{i}(l)}\left(z_{l}\right) d z_{0} \ldots d z_{k} \\
& =\int_{z_{0}=-\infty}^{\infty} \frac{1}{\sigma_{\mathrm{S}}} \varphi\left(\frac{z_{0}-m}{\sigma_{\mathrm{S}}}\right) \Phi^{k}\left(\frac{a-z_{0}}{t}\right) d z_{0},
\end{aligned}
$$

where $\varphi(\cdot)$ and $\Phi(\cdot)$ are, respectively, the density function and the cumulative distribution function of the (univariate) standard normal distribution. Similarly, the numerator is

$$
\begin{aligned}
E\left[F_{i, 1} ; \mathbf{D}_{i}\right]= & \int_{z_{0}=-\infty}^{\infty} \int_{z_{1}=-\infty}^{a-z_{0}} \ldots \int_{z_{k}=-\infty}^{a-z_{0}}\left(z_{0}+z_{1}\right) f_{\mu_{i}}\left(z_{0}\right) \prod_{l=1}^{k} f_{\hat{f}_{i}(l)}\left(z_{l}\right) d z_{0} \ldots d z_{k} \\
= & \int_{z_{0}=-\infty}^{\infty} z_{0} f_{\mu_{i}}\left(z_{0}\right) \Phi^{k}\left(\frac{a-z_{0}}{t}\right) d z_{0} \\
& +\int_{z_{0}=-\infty}^{\infty} f_{\mu_{i}}\left(z_{0}\right)\left[\int_{z_{1}=-\infty}^{a-z_{0}} z_{1} f_{\hat{f}_{i}(1)}\left(z_{1}\right) d z_{1}\right] \Phi^{k-1}\left(\frac{a-z_{0}}{t}\right) d z_{0} .
\end{aligned}
$$

Using results from Patel and Read (1982) about the mean of the truncated (one-dimensional) normal distribution, it follows that

$$
\int_{-\infty}^{a-z_{0}} z f_{\hat{f}_{i}(1)}(z) d z=-t \varphi\left(\frac{a-z_{0}}{t}\right)
$$

so we have

$$
E\left[F_{i, 1} ; \mathbf{D}_{i}\right]=\int_{-\infty}^{\infty}\left[z_{0} \Phi\left(\frac{a-z_{0}}{t}\right)-t \varphi\left(\frac{a-z_{0}}{t}\right)\right] \frac{1}{\sigma_{\mathrm{S}}} \varphi\left(\frac{z_{0}-m}{\sigma_{\mathrm{S}}}\right) \Phi^{k-1}\left(\frac{a-z_{0}}{t}\right) d z_{0} .
$$


Thus, we can express $E\left[F_{i, 1} \mid \mathbf{D}_{i}\right]$ as the quotient of two one-dimensional integrals. Using another result from Patel and Read (1982), we have

$$
\int_{-\infty}^{a-z_{0}} z^{2} f_{\hat{f}_{i}(1)}(z) d z=t^{2}\left[\Phi\left(\frac{a-z_{0}}{t}\right)-\frac{a-z_{0}}{t} \varphi\left(\frac{a-z_{0}}{t}\right)\right],
$$

so all other types of conditional expectations and categories of vectors can be treated in a similar fashion. Now define the three quantities

$$
S_{j}=\sum_{i=1}^{n} \sum_{r=1}^{k} E_{\boldsymbol{\theta}_{j}}\left[F_{i, r} \mid \mathbf{D}\right], \quad P_{j}=\sum_{i=1}^{n} \sum_{r=1}^{k} E_{\boldsymbol{\theta}_{j}}\left[F_{i, r}^{2} \mid \mathbf{D}\right], \quad Q_{j}=\sum_{i=1}^{n} \sum_{r=1}^{k} \sum_{r^{\prime} \neq r} E_{\boldsymbol{\theta}_{j}}\left[F_{i, r} F_{i, r^{\prime}} \mid \mathbf{D}\right]
$$

which, by the above, can be computed using one-dimensional numerical integrations. Since maximizing the log-likelihood function in Section B.4 is completely analogous to maximizing $Q$ here, we can use (38) and derive the following closed-form expressions for the maximizers in the M-step of the EM algorithm:

$$
\boldsymbol{\theta}_{j+1}=\left(m_{j+1}, \sigma_{\mathrm{S}, j+1}^{2}, t_{j+1}^{2}\right)=\underset{\boldsymbol{\theta}}{\arg \max } Q\left(\boldsymbol{\theta}, \boldsymbol{\theta}_{j}\right),
$$

where

$$
m_{j+1}=\frac{S_{j}}{n k}, \quad \sigma_{\mathrm{S}, j+1}^{2}=\frac{Q_{j}}{n k(k-1)}-\frac{S_{j}^{2}}{n^{2} k^{2}}, \quad t_{j+1}^{2}=\frac{P_{j}}{n k}-\frac{Q_{j}}{n k(k-1)} .
$$

Now we have all the necessary analytical results to run the EM algorithm, based on the 1-mutants data of stage I of the design. After 60 iterations of the algorithm, it converged to the values

$$
\hat{m}=-1.35, \quad \hat{\sigma}_{\mathrm{S}}^{2}=0.25, \quad \hat{t}^{2}=0.35 \text {. }
$$

B.6. Estimation based on multiple-site mutation data. To decompose the sum $t^{2}=\sigma_{\mathrm{R}}^{2}+\sigma_{\mathrm{N}}^{2}$ into its components, we use the multiple-site mutation data obtained during stage II of the design (see Section B.1). This data consists of 242 points (most of which are truncated), and it contains all the available information about the partition of $t^{2}$. Unlike the 1-mutant data discussed in Section B.5, the selection of these 242 points is biased-the corresponding sequences were carefully chosen based on the results of stage I to find highfitness variants. Being aware of this bias, we use this data only to decompose $t^{2}$ into its components.

Let $\mathbf{X}$ be the multivariate normal 242-vector corresponding to the data; as before, its mean vector and covariance matrix under any parameterization can be found using (6)-(8). Also let $\mathbf{y}$ be the 14-vector of the fully observed 14 fitness values (Table 3 in Wu et al., 1998), and let b be the constant 228-vector corresponding to the truncated remaining 228 coordinates (all the components of this vector are equal to $\left.\log _{10}(27.6 / 1.5)=1.264\right)$. As before, we need to find in the $(j+1)$ st iteration of the EM algorithm parameters $\boldsymbol{\theta}$ to maximize

$$
Q\left(\boldsymbol{\theta}, \boldsymbol{\theta}_{j}\right)=E_{\boldsymbol{\theta}_{j}}\left[l_{c}(\boldsymbol{\theta} ; \mathbf{X}) \mid \mathbf{y}, \mathbf{b}\right]
$$

Evaluating $Q$ numerically for fixed $\boldsymbol{\theta}$ involves complex integration, and finding the maximizer $\boldsymbol{\theta}_{j+1}$ is even harder. To overcome these difficulties, we used Monte Carlo (MC) integration and a grid search: in each iteration of the EM algorithm, we construct a grid for the parameters $\boldsymbol{\theta}$, evaluate $Q$ for each of its values (in a way to be discussed momentarily), and choose as $\boldsymbol{\theta}_{j+1}$ the maximizers of $Q$ on the grid.

To evaluate $Q$ for each of the grid points $\boldsymbol{\theta}$, we generated 3,000 realization of $l_{c}(\boldsymbol{\theta} ; \mathbf{X}) \mid \mathbf{y}, \mathbf{b}$ under the parameterization $\boldsymbol{\theta}_{j}$, and calculated their mean. Recall that only 14 components of the vector $\mathbf{X}$ are fully observed and that the remaining 228 are only known to lie below the value 1.264 . Without loss of generality, we let the known components of $\mathbf{X}$ be the first ones and partition $\mathbf{X}$ accordingly as $\mathbf{X}=\left(\mathbf{X}_{1}, \mathbf{X}_{2}\right)$, where $\mathbf{X}_{1}=\left(X_{1}, \ldots, X_{14}\right)$ and $\mathbf{X}_{2}=\left(X_{15}, \ldots, X_{242}\right)$. One way to generate a realization of $\mathbf{X} \mid \mathbf{y}, \mathbf{b}$ is to compute the conditional distribution of $\mathbf{X}_{2} \mid \mathbf{X}_{1}=\mathbf{y}$ (which can be done using (10)) and then keep generating realizations thereof until getting one in which all components are below 1.264. The problem with this approach is that there is an extremely low probability that all the components of $\mathbf{X}_{2} \mid \mathbf{X}_{1}=\mathbf{y}$ 
will be below 1.264, so a very large number of trials will be needed to generate a single realization. To overcome this difficulty, we used Gibbs sampling (Casella and George, 1992), also known as Markov chain Monte Carlo.

We start by generating independent realizations of $X_{15} \mid \mathbf{X}_{1}=\mathbf{y}$ until obtaining one below 1.264, and denote this realized value by $x_{15}^{(0)}$. Next, we generate independent realizations of $X_{16} \mid \mathbf{X}_{1}=\mathbf{y}, X_{15}=x_{15}^{(0)}$ until obtaining one below 1.264 , which we denote by $x_{16}^{(0)}$. We continue this way, until getting a realization $x_{242}^{(0)}$ of $X_{242} \mid \mathbf{X}_{1}=\mathbf{y}, X_{15}=x_{15}^{(0)}, \ldots, X_{241}=x_{241}^{(0)}$ that is also below 1.264. That was the "initialization" round. In round 1, we first generate realizations of $X_{15} \mid \mathbf{X}_{1}=\mathbf{y}, X_{16}=x_{16}^{(0)}, X_{17}=x_{17}^{(0)} \ldots, X_{242}=x_{242}^{(0)}$, and denote by $x_{15}^{(1)}$ the first to be below 1.264. The next step in round 1 is to generate $x_{16}^{(1)}$, a realization of $X_{16} \mid \mathbf{X}_{1}=\mathbf{y}, X_{15}=x_{15}^{(1)}, X_{17}=x_{17}^{(0)} \ldots, X_{242}=x_{242}^{(0)}$ below 1.264. We continue round 1 this way, and the last step in it is a realization $x_{242}^{(1)}$ of $X_{242} \mid \mathbf{X}_{1}=\mathbf{y}, X_{15}=x_{15}^{(1)}, \ldots, X_{241}=x_{241}^{(1)}$. More generally, the $k$ th step in round $j$ is the generation of $x_{14+k}^{(j)}$, a realization of $X_{14+k} \mid \mathbf{X}_{1}=\mathbf{y}, X_{15}=x_{15}^{(j)}, \ldots, X_{13+k}=$ $x_{13+k}^{(j)}, X_{15+k}=x_{15+k}^{(j-1)}, \ldots, X_{242}=x_{242}^{(j-1)}$ that is below 1.264. Then, the distribution of the vector in hand at the end of each round, namely, $\mathbf{x}^{(j)}=\left(\mathbf{y}, x_{15}^{(j)}, \ldots, x_{242}^{(j)}\right)$, converges to that $\mathbf{X} \mid \mathbf{y}, \mathbf{b}$ as $j$ tends to infinity. Thus, for large $j$, we may take $\mathbf{x}^{(j)}$ as a single realization of the desired vector.

Rather than repeating the above full process for each of the 3,000 realizations used for each of the grid points in each of the EM algorithm iterations, we take as the 3,000 realizations the values of $\mathbf{x}^{(B)}, \mathbf{x}^{(B+\delta)}, \mathbf{x}^{(B+2 \delta)}, \ldots, \mathbf{x}^{(B+2999 \delta)}$ in a single run of the process, for some choice of $B$ and $\delta$. Although the realized vectors are dependent, the linearity of the expectation operators guarantees that their mean (or the mean of functionals thereof) converges to the right quantity. More specifically, we took $B=2,000$ and $\delta=3$.

Running the EM algorithm 100 iterations this way, we arrived at the results $\hat{\sigma}_{\mathrm{R}}^{2}=0.18$ and $\hat{\sigma}_{\mathrm{N}}^{2}=0.14$. Notice that the sum of these two values, 0.32, is remarkably close to $t^{2}=0.35$, obtained in Section B.5 from a totally different dataset.

B.7. Testing for goodness-of-fit. Given the above estimated parameters, we can generate by computer many realizations of the fitness values of all 1-mutants and find empirically the distribution of $F^{(1)}=$ $\max \left\{F_{s}, s \in \mathscr{S}_{1}\right\}$. The $K_{\mathrm{d}}$ score of the best 1-mutant found in stage I by Wu et al. (1998) is 2.2, which, when transformed to our model, corresponds to a fitness of $\log (27.6 / 2.2)=1.098$. One may reject our three-parameter model in Section B.5 on grounds of poor fit if the number 1.098 lies far at one of the tails of the distribution of $F^{(1)}$.

Based on 100,000 realizations of the 1-mutants landscape, we found that 1.098 coincides almost exactly with the median of $F^{(1)}$; more precisely, the number 1.098 corresponds to the 0.5119 quantile of the distribution of $F^{(1)}$. Thus, the result of this test provides no evidence to reject the three-parameter model in Section B.5. We did not attempt a goodness-of-fit test for the full four-parameter model because of the very small amount and very biased nature (i.e., only the most fit variants were considered) of the multiple-site mutation data.

\section{Slepian's inequality}

The following is based on Section 2.1 of Tong (1980). Let

$$
\left(X_{1}, \ldots, X_{n}\right) \sim N(\boldsymbol{\mu}, \boldsymbol{\Sigma}), \quad \text { and } \quad\left(X_{1}^{\prime}, \ldots, X_{n}^{\prime}\right) \sim N\left(\boldsymbol{\mu}, \boldsymbol{\Sigma}^{\prime}\right),
$$

where $\boldsymbol{\Sigma}=\left(\sigma_{i j}\right)$ and $\boldsymbol{\Sigma}^{\prime}=\left(\sigma_{i j}^{\prime}\right)$ satisfy

$$
\sigma_{i i}=\sigma_{i i}^{\prime}, i=1, \ldots, n, \quad \text { and } \quad \sigma_{i j} \leq \sigma_{i j}^{\prime}, i \neq j .
$$

Then, for all $a_{1}, \ldots, a_{n} \in \mathbb{R}$,

$$
P\left(X_{i} \leq a_{i}, i=1, \ldots, n\right) \leq P\left(X_{i}^{\prime} \leq a_{i}, i=1, \ldots, n\right) .
$$


By choosing $a_{i}=a$, we get the stochastic domination

$$
P\left(\max X_{i} \leq a\right) \leq P\left(\max X_{i}^{\prime} \leq a\right) .
$$

\section{Convergence of the maximal normal to a Gumbel}

The following is based on Theorem 1.5.3 of Leadbetter et al. (1980). A random variable $X$ is said to have a Gumbel distribution with parameters $\alpha$ and $\beta$ if it has the distribution function

$$
P(X<x)=\exp \left\{-e^{-\alpha(x-\beta)}\right\}, \quad x \in \mathbb{R},
$$

in which case

$$
E[X]=\beta+\frac{\gamma}{\alpha},
$$

where $\gamma=0.5772 \ldots$ is Euler's constant. Let $X_{1}, X_{2}, \ldots$ be a sequence of standard normal random variables, and define $M_{n}=\max \left\{X_{1}, \ldots, X_{n}\right\}$. Then, under the proper normalization, $M_{n}$ converges in distribution to a Gumbel random variable with parameters $\alpha=1$ and $\beta=0$ :

$$
P\left(a_{n}\left(M_{n}-b_{n}\right) \leq x\right) \rightarrow \exp \left\{-e^{-x}\right\} \quad \text { as } n \rightarrow \infty, \quad x \in \mathbb{R}
$$

where

$$
a_{n}=\sqrt{2 \log n} \quad \text { and } \quad b_{n}=\sqrt{2 \log n}-\frac{\log \log n+\log 4 \pi}{2 \sqrt{2 \log n}} .
$$

\section{E. Majorization}

The following is based on Tong (1988). Let $\mathbf{k}=\left(k_{i}\right)$ and $\mathbf{k}^{\prime}=\left(k_{i}^{\prime}\right)$ be two real $n$-vectors, and let their ordered components be $k_{[1]} \geq \cdots \geq k_{[n]}$ and $k_{[1]}^{\prime} \geq \cdots \geq k_{[n]}^{\prime}$. The vector $\mathbf{k}$ is said to majorize the vector $\mathbf{k}^{\prime}$ if

$$
\sum_{i=1}^{l} k_{[i]} \geq \sum_{i=1}^{l} k_{[i]}^{\prime} \quad \text { for } l=1, \ldots, n .
$$

Thus, the concept of majorization concerns the comparison of degree of diversity among the components between the vectors $\mathbf{k}$ and $\mathbf{k}^{\prime}$.

Let $\mathbf{X}=\left(X_{j}\right)$ be a multivariate normal vector having a constant mean vector $\boldsymbol{\mu}=(\mu, \ldots, \mu)$ and covariance matrix $\boldsymbol{\Sigma}(\mathbf{k})$ of the block form

$$
\boldsymbol{\Sigma}(\mathbf{k})=\left(\begin{array}{cccc}
\boldsymbol{\Sigma}_{1} & \mathbf{0} & \cdots & \mathbf{0} \\
\mathbf{0} & \boldsymbol{\Sigma}_{2} & \cdots & \mathbf{0} \\
& \cdots & & \\
\mathbf{0} & \mathbf{0} & \cdots & \boldsymbol{\Sigma}_{n}
\end{array}\right),
$$

where $\Sigma_{i}$ is a $k_{i} \times k_{i}$ positive-definite matrix having the form

$$
\boldsymbol{\Sigma}_{i}=\left(\begin{array}{cccc}
a & b & \cdots & b \\
b & a & \cdots & b \\
& \cdots & & \\
b & b & \cdots & a
\end{array}\right)
$$

for two real numbers $a$ and $b$. Let $\mathbf{X}^{\prime}=\left(X_{j}^{\prime}\right)$ be another multivariate normal vector defined similarly, having the same mean vector $\boldsymbol{\mu}$ and the same underlying $a$ and $b$, but with the vector $\mathbf{k}^{\prime}$ replacing $\mathbf{k}$. Then, if $\mathbf{k}$ majorizes $\mathbf{k}^{\prime}$, we have

$$
P\left(\max X_{j}>x\right) \leq P\left(\max X_{j}^{\prime}>x\right), \quad x \in \mathbb{R} ;
$$

i.e., $\max X_{j}^{\prime}$ stochastically dominates $\max X_{j}$. 


\section{F. Total-covariance minimization}

In this appendix, we formulate the total-covariance minimization problem corresponding to (22) as an integer program and solve it. Without loss of generality, we label the 19 substitution residues at each site by $1, \ldots, 19$. Let $x_{s, i, r}$ be a binary decision variable indicating whether sequence $s$ of the design includes in site $i$ residue $r(s=1, \ldots, K, i=1, \ldots, n, r=1, \ldots, 19)$. Then, for two sequences $s, s^{\prime} \in \mathscr{S}$, we have that (see (5))

$$
\left|M_{1}\left(s, s^{\prime}\right)\right|=\sum_{i=1}^{n}\left(\sum_{r=1}^{19} x_{s, i, r}\right)\left(\sum_{r^{\prime}=1}^{19} x_{s^{\prime}, i, r^{\prime}}\right) \quad \text { and } \quad\left|M_{2}\left(s, s^{\prime}\right)\right|=\sum_{i=1}^{n} \sum_{r=1}^{19} x_{s, i, r} x_{s^{\prime}, i, r} .
$$

Therefore, (8) implies that for $s \neq s^{\prime}$,

$$
\operatorname{Cov}\left(F_{S}, F_{s^{\prime}}\right)=\sigma_{\mathrm{S}}^{2} \sum_{i=1}^{n}\left(\sum_{r=1}^{19} x_{s, i, r}\right)\left(\sum_{r^{\prime}=1}^{19} x_{s^{\prime}, i, r^{\prime}}\right)+\sigma_{\mathrm{R}}^{2} \sum_{i=1}^{n} \sum_{r=1}^{19} x_{s, i, r} x_{s^{\prime}, i, r} .
$$

To express the fitness variance of a sequence $s \in \mathscr{S}_{p}$, we note that $p=d(s, \hat{s})=\left|M_{1}(s, s)\right|=\left|M_{2}(s, s)\right|$, and thus (7) and (41) give

$$
\operatorname{Var}\left(F_{S}\right)=\sigma_{\mathrm{S}}^{2} \sum_{i=1}^{n}\left(\sum_{r=1}^{19} x_{s, i, r}\right)\left(\sum_{r^{\prime}=1}^{19} x_{s, i, r^{\prime}}\right)+\sigma_{\mathrm{R}}^{2} \sum_{i=1}^{n} \sum_{r=1}^{19} x_{s, i, r} x_{s, i, r}+\sigma_{\mathrm{N}}^{2} .
$$

By interchanging the order of summation, we may write the total covariance $\tau(D)$ in $(21)$ as

$$
\begin{aligned}
\tau(D) & =\sigma_{\mathrm{S}}^{2} \sum_{s, s^{\prime}} \sum_{i}\left(\sum_{r} x_{s, i, r}\right)\left(\sum_{r^{\prime}} x_{s^{\prime}, i, r^{\prime}}\right)+\sigma_{\mathrm{R}}^{2} \sum_{s, s^{\prime}} \sum_{i} \sum_{r} x_{s, i, r} x_{s^{\prime}, i, r}+K \sigma_{\mathrm{N}}^{2} \\
& =\sigma_{\mathrm{S}}^{2} \sum_{i}\left(\sum_{s, r} x_{s, i, r}\right)^{2}+\sigma_{\mathrm{R}}^{2} \sum_{i, r}\left(\sum_{s} x_{s, i, r}\right)^{2}+K \sigma_{\mathrm{N}}^{2}
\end{aligned}
$$

The term $K \sigma_{\mathrm{N}}^{2}$ in the last expression is constant and can be ignored for minimization purposes. The total-covariance minimization problem of a design $D \in \mathscr{D}_{K}(p)$ can be formulated therefore as

$$
\begin{array}{ll}
\underset{x_{s, i, r}}{\operatorname{minimize}} & \sigma_{\mathrm{S}}^{2} \sum_{i}\left(\sum_{s, r} x_{s, i, r}\right)^{2}+\sigma_{\mathrm{R}}^{2} \sum_{i, r}\left(\sum_{s} x_{s, i, r}\right)^{2} \\
\text { s.t. } \quad & \sum_{r} x_{s, i, r} \leq 1 \quad \text { for all } s, i, \\
& \sum_{i, r} x_{s, i, r}=p \quad \text { for all } s, \\
& \sum_{i, r} x_{s, i, r} x_{s^{\prime}, i, r} \leq p-1 \quad \text { for all } s \neq s^{\prime}, \\
& x_{s, i, r} \in\{0,1\} .
\end{array}
$$

The first set of constraints guarantees that a sequence will not include more than one residue at each site; the second guarantees that we consider only $p$-mutants; the third makes sure that the sequences are distinct.

We now solve the integer program (42) and show how to build, for each $p$, an ordered list of $p$-mutants having the property that the first $K$ elements thereof constitute a covariance-minimizing design in $\mathscr{D}_{K}(p)$. 
We first need the following result: the optimal solutions of the integer program

$$
\operatorname{minimize} \sum_{i=1}^{n} y_{i}^{2} \quad \text { s.t. } \sum_{i=1}^{n} y_{i}=B, \quad y_{i} \in\{0,1,2, \ldots\}
$$

are exactly those natural-valued $\left\{y_{i}\right\}$ whose sum is $B$ and that satisfy

$$
\left|y_{i}-y_{j}\right| \leq 1 \quad \text { for all } i, j \text {. }
$$

The proof is straightforward: given a feasible solution $y_{1}, \ldots, y_{n}$ with $y_{i}-y_{j} \geq 2$ for some $i \neq j$, subtract 1 from $y_{i}$ and add 1 to $y_{j}$. The resulting solution is still feasible, and the objective function decreases by at least 2 . The only case where we cannot do this is when the $y_{i}$ are "as equal as possible," i.e., when (44) holds.

Returning to our total-covariance minimization problem, we define

$$
y_{i}=\sum_{s, r} x_{s, i, r} \quad \text { and } \quad z_{i, r}=\sum_{s} x_{s, i, r},
$$

so that the objective function can now be written as

$$
\sigma_{\mathrm{S}}^{2} \sum_{i} y_{i}^{2}+\sigma_{\mathrm{R}}^{2} \sum_{i, r} z_{i, r}^{2}
$$

From the second set of constraints of the integer program (42), we have that

$$
\sum_{i} y_{i}=\sum_{i, r} z_{i, r}=K p
$$

and we think of $K p$ as the constant $B$ in (43). Now suppose that we find a feasible solution $\left\{x_{s, i, r}^{*}\right\}$ such that both the induced $y_{i}$ and the induced $z_{i, r}$ are as nearly equal as possible. This solution will minimize both $\sum_{i} y_{i}^{2}$ and $\sum_{i, r} z_{i, r}^{2}$ and will therefore minimize also their positively weighted sum in (45).

All that is left to do is to find such a solution $\left\{x_{s, i, r}^{*}\right\}$. This is more difficult to describe than to do, so we illustrate that by an example for the case $n=6, p=2$. We now provide a list of sequences enjoying a "monotonicity" property, by which the first $K$ sequences constitute an optimal solution for the IP (42) when $D \in \mathscr{D}_{K}(2)$, i.e., the induced $y_{i}$ and $z_{i, r}$ are as nearly equal as possible at any point along the list.

We represent each sequence as a 6-vector whose $i$ th component represents the $i$ th residue. Without loss of generality, we label the wild-type residue at each of the six sites by the symbol " 0 ," and each of the 19 possible substitution by $1, \ldots, 19$.

As the first $n / p=3$ sequences, we may take

$$
(1,1,0,0,0,0), \quad(0,0,1,1,0,0), \quad(0,0,0,0,1,1) .
$$

This choice yields (here $K=3$ )

$$
y_{1}=y_{2}=\ldots=y_{6}=1
$$

and

$$
z_{1,1}=z_{2,1}=\ldots=z_{6,1}=1 ; \quad z_{i, r}=0 \text { for } i \neq 1 .
$$

Indeed, both the $y_{i}$ 's and the $z_{i, r}$ 's are "as equal as possible." As the next $n / p=3$ sequences we may have

$$
(2,2,0,0,0,0), \quad(0,0,2,2,0,0), \quad(0,0,0,0,2,2),
$$

which gives (now for $K=6$ )

$$
y_{1}=y_{2}=\ldots=y_{6}=2
$$


and

$$
z_{1,1}=z_{2,1}=\ldots=z_{6,1}=1 ; \quad z_{1,2}=z_{2,2}=\ldots=z_{6,2}=1 ; \quad z_{i, r}=0 \text { for } i \neq 1,2 .
$$

Again, the $y_{i}$ 's and the $z_{i, r}$ 's are "as equal as possible." Continuing this way, we end up with the following sequences as the first $19 n / p=57$ ones:

$$
\begin{array}{ccc}
(1,1,0,0,0,0) & (0,0,1,1,0,0) & (0,0,0,0,1,1) \\
(2,2,0,0,0,0) & (0,0,2,2,0,0) & (0,0,0,0,2,2) \\
& \vdots & \\
(19,19,0,0,0,0) & (0,0,19,19,0,0) & (0,0,0,0,19,19) .
\end{array}
$$

The resulting $y_{i}$ 's and $z_{i, r}$ 's are now (here $K=57$ )

$$
y_{1}=y_{2}=\ldots=y_{6}=19 \text { and } z_{i, r}=1 \text { for all } i, r .
$$

As the next $19 n / p=57$ sequences we may choose

$$
\begin{array}{ccc}
(1,2,0,0,0,0) & (0,0,1,2,0,0) & (0,0,0,0,1,2) \\
(2,3,0,0,0,0) & (0,0,2,3,0,0) & (0,0,0,0,2,3) \\
& \vdots & \\
(19,1,0,0,0,0) & (0,0,19,1,0,0) & (0,0,0,0,19,1),
\end{array}
$$

which gives (now for $K=2 \cdot 19 n / p=114$ )

$$
y_{1}=y_{2}=\ldots=y_{6}=38 \text { and } z_{i, r}=2 \text { for all } i, r .
$$

The next $19 n / p=57$ sequences may be chosen to be

$$
\begin{array}{ccc}
(1,3,0,0,0,0) & (0,0,1,3,0,0) & (0,0,0,0,1,3) \\
(2,4,0,0,0,0) & (0,0,2,4,0,0) & (0,0,0,0,2,4) \\
& \vdots & \\
(19,2,0,0,0,0) & (0,0,19,2,0,0) & (0,0,0,0,19,2),
\end{array}
$$

and we can continue creating 16 more sets of $19 n / p=57$ sequences in this way, resulting in a total of $K=19 \cdot 19 n / p=1,083$ sequences. The last of these sets will be

$$
\begin{array}{ccc}
(1,19,0,0,0,0) & (0,0,1,19,0,0) & (0,0,0,0,1,19) \\
(2,1,0,0,0,0) & (0,0,2,1,0,0) & (0,0,0,0,2,1) \\
& \vdots & \\
(19,18,0,0,0,0) & (0,0,19,18,0,0) & (0,0,0,0,19,18) .
\end{array}
$$

Another set of $19 \cdot 19 n / p=1,083$ sequences can be formed by mutating at nonadjacent sites along the sequence. These may be, for example,

$$
\begin{array}{ccc}
(1,0,0,1,0,0) & (0,1,0,0,1,0) & (0,0,1,0,0,1) \\
(2,0,0,2,0,0) & (0,2,0,0,2,0) & (0,0,2,0,0,2) \\
& \vdots & \\
(19,0,0,19,0,0) & (0,19,0,0,19,0) & (0,0,19,0,0,19) \\
& \vdots & \\
(1,0,0,19,0,0) & (0,1,0,0,19,0) & (0,0,1,0,0,19) \\
(2,0,0,1,0,0) & (0,2,0,0,1,0) & (0,0,2,0,0,1) \\
& \vdots & \\
(19,0,0,18,0,0) & (0,19,0,0,18,0) & (0,0,19,0,0,18)
\end{array}
$$


Additional sets of $19 \cdot 19 n / p=1,083$ sequences can be created this way, by changing the location of the mutated sites. This methods results in a total of $K=19^{2} n^{2} / p=6,498$ sequences, ordered as a covariance-minimizing design.

Note that just as in the 1-mutants optimization problem (19), the optimal solutions of the integer program in (42) are independent of the landscape parameters $m, \sigma_{\mathrm{S}}^{2}, \sigma_{\mathrm{R}}^{2}$, and $\sigma_{\mathrm{N}}^{2}$.

\section{G. The Sudakov-Fernique inequality}

The following is based on Theorem 2.9 in Adler (1990). Let $\left\{X_{i}, i \in I\right\}$ and $\left\{X_{i}^{\prime}, i \in I\right\}$ be two zero-mean Gaussian processes on the same countable index set $I$, satisfying

$$
E\left(X_{i}-X_{j}\right)^{2} \leq E\left(X_{i}^{\prime}-X_{j}^{\prime}\right)^{2} \quad \text { for all } i, j \in I .
$$

Then $E \sup _{i \in I} X_{i} \leq E \sup _{i \in I} X_{i}^{\prime}$.

\section{REFERENCES}

Adler, R.J. 1990. An Introduction to Continuity, Extrema and Related Topics for General Gaussian Processes, Institute of Mathematical Statistics, Hayward, California.

Aita, T., and Husimi, Y. 2000a. Adaptive walks by the fittest among finite random mutants on a Mt. Fuji-type fitness landscape-II. Effects of small non-additivity. J. Math. Biol. 41, 207-231.

Aita, T., and Husimi, Y. 2000b. Theory of evolutionary molecular engineering through simultaneous accumulation of advantageous mutations. J. Theor. Biol. 207, 543-556.

Aita, T., Iwakura, M., and Husimi, Y. 2001. A cross-section of the fitness landscape of dihydrofolate reductase. Protein Eng. 14 (9), 633-638.

Aita, T., Uchiyama, H., Inaoka, T., Nakajima, M., Kokubo, T., and Husimi, Y. 2000. Analysis of a local fitness landscape with a model of the rough Mt. Fuji-type landscape: Application to prolyl endopeptidase and thermolysin. Biopolymers 54, 64-79.

Altamirano, M.M., Blackburn, J.M., Aguayo, C., and Fersht, A.R. 2000. Directed evolution of new catalytic activity using the $\alpha / \beta$-barrel scaffold. Nature 403, 617-622.

Anthony-Cahill, S.J., and Magliery, T.J. 2002. Expanding the natural repertoire of protein structure and function. Current Pharmaceutical Biotechnology 3, 299-315.

BCC Research. 2003. Protein drugs are the next blockbusters. Industries in Transition 31 (5).

Casella, G., and George, E.I. 1992. Explaining the Gibbs sampler. Am. Statistician 46 (3), 167-174.

Clark, C.E. 1961. The greatest of a finite set of random variables. Operations Res. 9, 145-162.

Cover, T.M., and Thomas, J.A. 1991. Elements of Information Theory, John Wiley, New York.

Cox, D., and Oakes, D. 1984. Analysis of Survival Data, Chapman and Hall, London.

Dempster, A.P., Laird, N.M., and Rubin, D.B. 1977. Maximum likelihood from incomplete data via the EM algorithm. J. Royal Stat. Soc. Series B 39, 1-38.

Derrida, B. 1981. Random-energy model: An exactly solvable model of disordered systems. Phys. Rev. B 24 (5), 2613-2626.

Eigen, M., McCaskill, J., and Schuster, P. 1989. The molecular quasi-species. Adv. Chem. Phys. 75, 149-263.

Fontana, W., and Schuster, P. 1998. Continuity in evolution: On the nature of transitions. Science 280, 1451-1455.

Fox, R., Roy A., Givindarajan, S., Minshull, J., Gustafsson, C., Jones, J.T., and Emig, R. 2003. Optimizing the search algorithm for protein engineering by directed evolution. Protein Eng. 16 (8), 589-597.

Goldsby, R.A., Kindt, T.J., and Osborne, B. 2000. Immunology, 4th ed., W.H. Freeman, San Francisco.

Govindarajan, S., and Goldstein, R.A. 1997. Evolution of model proteins on a foldability landscape. Prot. Struct. Funct. Genet. 29, 461-466.

Hamming, R.W. 1950. Error detecting and error correcting codes. Bell Systems Tech. J. 29, 147-160.

Johnson, D.B., and Mizoguchi, T. 1978. Selecting the $K$ th element in $X+Y$ and $X_{1}+X_{2}+\ldots+X_{m}$. SIAMJ. Comput. 7, 147-153.

Kauffman, S.A., and Levin, S. 1987. Towards a general theory of adaptive walks on rugged landscapes. J. Theoret. Biol. 128, 11-45.

Kella, O. 1986. On the distribution of the maximum of bivariate normal random variables with general means and variances. Communications in Statistics: Theory and Methods 15 (11), 3265-3276.

Leadbetter, M.R., Lindgren, G., and Holger, R. 1980. Extremes and Related Properties of Random Sequences and Processes, Springer-Verlag, New York. 
Maynard Smith, J. 1970. Natural selection and the concept of a protein space. Nature 225, 563-564.

McLachlan, G.J., and Krishnan, T. 1997. The EM Algorithm and Extensions, John Wiley, New York.

Patel, J., and Read, C. 1982. Handbook of the Normal Distribution, Marcel Dekker, New York.

Perelson, A.S., and Macken, C.A. 1994. Protein evolution on partially correlated landscapes. Technical Report 94-11060, Santa Fe Institute.

Stryer, L. 1995. Biochemistry, W.H. Freeman, San Francisco.

Tong, Y.L. 1980. Probability Inequalities in Multivariate Distribution, Academic Press, New York.

Tong, Y.L. 1988. Some majorization inequalities in multivariate statistical analysis. SIAM Review 30 (4), $602-622$.

Tong, Y.L. 1990. The Multivariate Normal Distribution, Springer-Verlag, New York.

Voigt, C.A., Kauffman, S., and Wang, Z.-G. 2001. Rational evolutionary design: The theory of in vitro protein evolution. Adv. Protein Chem. 55, 79-160.

Walsh, G. 2002. Proteins: Biochemistry and Biotechnology, John Wiley, New York.

Weinberger, E.D. 1990. Correlated and uncorrelated fitness landscapes and how to tell the difference. Biol. Cybern. 63, 325-336.

Wells, J. 1990. Additivity of mutational effects in proteins. Biochemistry 29, 8509-8517.

Wright, S.G. 1932. The roles of mutation, inbreeding, crossbreeding and selection in evolution. Proc. 6th Int. Congress on Genetics 1, 356-366.

Wu, H., Beuerlein, G., Nie, Y., Smith, H., Lee, B., Hensler, M., Huse, W., and Watkins, J. 1998. Stepwise in vitro affinity maturation of Vitaxin, an $\alpha_{\mathrm{v}} \beta_{3}$-specific humanized mAb. Proc. Natl. Acad. Sci. 95, 6037-6042.

Address correspondence to: Lawrence M. Wein

Graduate School of Business 518 Memorial Way Stanford University Stanford, CA 94305

E-mail: lwein@stanford.edu 